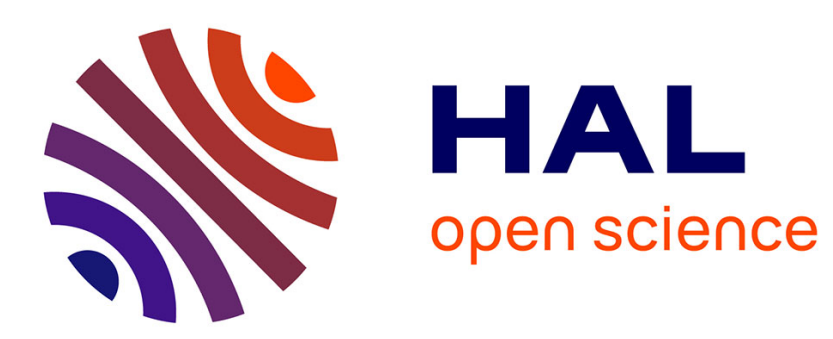

\title{
On the origins of the Finnis-Sinclair potentials
}

Olivier Hardouin Duparc

\section{To cite this version:}

Olivier Hardouin Duparc. On the origins of the Finnis-Sinclair potentials. Philosophical Magazine, 2009, 89 (34-36), pp.3117-3131. 10.1080/14786430903292423 . hal-00541685

\section{HAL Id: hal-00541685 \\ https://hal.science/hal-00541685}

Submitted on 1 Dec 2010

HAL is a multi-disciplinary open access archive for the deposit and dissemination of scientific research documents, whether they are published or not. The documents may come from teaching and research institutions in France or abroad, or from public or private research centers.
L'archive ouverte pluridisciplinaire HAL, est destinée au dépôt et à la diffusion de documents scientifiques de niveau recherche, publiés ou non, émanant des établissements d'enseignement et de recherche français ou étrangers, des laboratoires publics ou privés. 


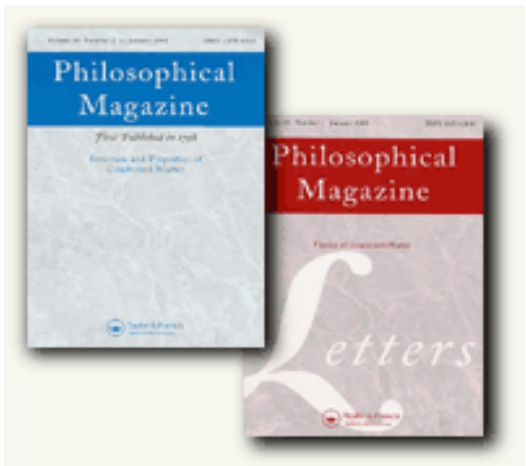

\section{On the origins of the Finnis-Sinclair potentials}

\begin{tabular}{|r|l|}
\hline Journal: & Philosophical Magazine \& Philosophical Magazine Letters \\
\hline Manuscript ID: & TPHM-09-May-0226.R2 \\
\hline Journal Selection: & Philosophical Magazine \\
\hline Date Submitted by the \\
Author: & 06-Aug-2009 \\
\hline Complete List of Authors: & $\begin{array}{l}\text { HARDOUIN DUPARC, Olivier; LSI, CEA-X-CNRS, UMR 7642, Ecole } \\
\text { Polytechnique }\end{array}$ \\
\hline Keywords: & $\begin{array}{l}\text { atomistic simulation, band theory, elasticity, molecular dynamics, } \\
\text { point defects, semiconductors, tight-binding, transition metals }\end{array}$ \\
\hline Keywords (user supplied): & Finnis-Sinclair potentials, methof of moments, history of sciences \\
\hline &
\end{tabular}

\section{s ScholarONE" \\ Manuscript Central}


I trace back the origins of the famous Finnis-Sinclair potentials. These potentials mimic the results of tight binding theory through their use of the square root embedding function. From the tentative beginnings of tight binding in the 1930s up to 1984 or so, some of the famous names involved are Bloch, Seitz, Montroll, Friedel, Cyrot-Lackmann, Ducastelle, to name just a few. The application of the method of moments to the description of densities of states and its connexion to the physics of closed paths linking nearest neighbours interacting atoms helped to formalize Friedel's rectangular band model for the $d$ electrons in transition metals. Extension from perfectly periodic structures to defective ones could not be but a slow process due to the change of paradigm for solid state scientists and to the necessary caution to be paid to self-consistency. The British scientists school also contributed significantly in the $80 \mathrm{~s}$. Computer progress and pragmatism helped to go from mainly analytical developments to numerical experiments (another change of paradigm). I also digress on various not so well known historical points of interest to this story.

Keywords: Finnis-Sinclair Potentials; tight binding; LCAO; method of moments; second moment approximation; transition metals; semiconductors; Cauchy relations; embedded atom method; molecular dynamics; history of science

\section{Introduction}

Without going back to ancient Greek models (see [1]), one can say that pair potentials proved to be surprisingly efficient, at least as a first approximation, for metals and ionic crystals since Mie (1903), Grüneisen (1911-1912), Born (with Landé 1918, with Mayer 1932). Yet the consideration that metals are mainly governed by electrons moving more or less freely (Riecke, Drude, Lorentz, Thomson [Clausius-Boltzmann gas], Sommerfeld [Fermi gas] 1928) as in a gigantic molecule which should be treated through the Schrödinger equation (not to mention Dirac's equation) rapidly raised questions. Solving the Schrödinger equation for a large number of atoms in a condensed matter state, however, implies a lot of numerical work (now computer work) which restricts the size of the systems which can be considered. Most physicists have always tried to use, as much as possible, simple models endorsed with some physically interpretable parameters which can be varied at will (for numerical experimentation) and/or fitted versus trustable data (experimental and/or ab initio, when reliability is warranted) for real materials. Simplicity, for understanding, versus realism,

\footnotetext{
* Corresponding author. Email: olivier.hardouinduparc@ polytechnique.edu
} 
depending on the (complexity of the) question to be addressed: This depends on the conceptual tools, together with the numerical tools, that the physicist may use and master (e.g. irrational numbers and logarithms on one side, and computers on the other side). Conceptual tools for understanding evolve with numerical tools (and technology), and they also evolve in the sense that they may seem odd at first sight and slowly become natural once one gets used to them. They subsequently can be improved. It is this slow evolutionary process that I see at work in what led to what are now called the "Finnis-Sinclair potentials." My plan will simply follow history, starting in the 30s up to the 80s. I put in appendices some lateral information which I believe is important and not too well-known. I otherwise assume that the reader is acquainted with classical texts such as [2-8].

\section{The Thirties; first steps, nullified by sensible hesitations}

In 1935, naturally impressed by the recent breakthrough of Wigner and Seitz (1933, [9]) who had been able to account "quantitatively for the cohesive forces in a typical metal (sodium)," Mott attempted to "give a qualitative discussion, based on the same theory, of some properties of the transition metals" and in particular of the elements nickel and palladium. He considered the outermost $s$ electrons as responsible for the cohesion (and the $d$ electrons as responsible for the magnetic properties) and he estimated to 0.6 the number of $s$ electrons per atom for nickel. In a clever way, he concluded that nickel must have a binding energy greater than that of copper, which agrees with experimental data. Apart from nickel, palladium and platinum, the binding energy of transition metals was not considered and the rest of his article was mainly concerned with the discussion of magnetic susceptibilities and electrical conductivities [10].

In 1936, H. Fröhlich endorsed this analysis in his textbook for all transition metals in a forcedly odd way ( $\$ 30$ of [11]) while Mott and Jones wrote that as far as transition metals are concerned, "no calculations of the binding forces in these metals have yet been made" (sic, Chapt. IV, § 3.3 of [12]).

In 1937, in the second of a set of three highly pedagogical articles, Seitz and Johnson proposed that "as long as the electrons fill depressed zones [the lower states of the bands] we may expect the binding to increase, but when levels which are higher than the atomic states are filled, we may expect the binding to decrease. That is, we expect the binding energy in one of the long periods $[3 d, 4 d, 5 d]$ to increase with atomic number at first, reach a maximum and then decrease again.” [13]. This simple tight-binding picture naturally implied a number 
of approximations they perfectly knew about and explained in their series of articles. Seitz only briefly alluded to this scheme in his 1940 book but he re-expressed it, more clearly, in 1955 with Wigner in the first volume of the Seitz and Turnbull's Solid State Physics series, stating that in the case of separated $p$ or $d$ bands, "the bands are centered essentially at the value of the atomic level as long as the wave functions are assumed to be composed of atomic functions in the manner [of the tight binding approximation]. The binding is largest for the first electron in each shell in this approximation, is zero for the electron in the middle of the shell, and is negative for the electrons in the second half of the shell. This means that the cohesive energy should attain its maximum near the center of the shell and decrease toward either end." And they provided the reader with a figure of the experimental cohesive energy of the condensed state as a function of the atomic number [14]. This seems to have gone rather unnoticed.

Also in the US, however (and even in the same State of New York), Slater wrote in 1936 that the electrons in the incomplete atomic $d$ shells can be responsible for magnetism but "do not take part in the cohesion," "cannot be engaged in cohesion" [15]. He also wrote that "Overlapping the band of $3 d$ electrons is another band, arising from the $4 s$ level of the atom, with interactions with the $4 p$ and other higher levels. (...) Electrons in this band act like free electrons (...) they contribute strongly to the cohesion." Slater was thus threatening the principle of LCAO calculations that neglected excited atomic states with higher energies but larger overlap and he developed in 1937 a model where the trial wave functions are expanded in terms of plane waves within spheres centred on the nuclei [16]. This celebrated muffin-tin model involves complex calculations the results of which were not easy to interpret (see [17$18])$.

At the end of the thirties, just before the Second World War broke out, discussions about magnetism at an international conference in Strasbourg simply questioned the possibility that $d$ electrons could form bands at all [19-20].

\section{The Sixties; the time of Jacques Friedel and his $d$ band model}

After the War, matters settled down, and in 1964, in his Institute of Metals Lecture, J. Friedel independently re-developed a tight-binding analysis of transition metals concentrating on the $d$ band only, in a simpler and more precise way [21]. In this Lecture, Friedel introduced his famous rectangular $d$ band model: "For all reasonable forms $n_{d}(E)$ of the $d$ band, one finds much the same parabolic variation as when $n_{d}=$ const." He then provided the reader with an 
equation which does give the parabolic variation of the atomic cohesive energy (with respect to isolated, free atoms, the sublimation energy) with the number of $d$ electrons per atom. (The direct proportionality to the electronic hopping integral between first neighbours $\beta$ was also clear but the proportionality to the square root of the coordination number of the structure, obviously a secondary point at that time, was missed in the equation simply because the width of the $d$ band was taken as $2|\beta|$, instead of $\sqrt{12}_{12}{\sqrt{z_{c}}}_{\mid}|\beta|$ as it should be for the rectangular $d$-band where $\mathrm{z}_{\mathrm{c}}$ is the coordination number: the number of nearest neighbours per atom. Note that for the real $d$-band, the width is of the order of $2 z_{c}|\beta|$. For the Gaussian $d$-band, the width (support) is infinite and one defines instead the standard deviation $\sigma=\sqrt{ }$ var $=\sqrt{ } \mu_{2}$ which equals $\sqrt{ }_{\mathrm{c}}|\beta|$. See Appendix A).

Françoise Cyrot, née Lackmann, substantiated her thesis director's assertion about "all reasonable forms $n_{d}(E)$ " in her $\mathrm{PhD}$ thesis (and in [22], in French). For that, she developed the integrals $n_{d}\left(E_{F}\right)\left[=\int{ }^{E_{F}} n_{d}(E) d E\right]$ and $E_{\mathrm{B}}\left(E_{F}\right)\left[=\int{ }^{E_{F}} E n_{d}(E) d E\right]$ for various forms of $n_{d}(E)$ and numerically plotted the latter versus the former. (Only for the rectangular model can one directly get an analytical formula showing a strict, shifted, parabolic dependence $a\left[n_{d}\left(E_{F}\right)-5\right]^{2}$.) She used the mathematical formalism of moments (see Appendix A) to approximate the density of states (DOS) of the electrons in transition metals for two reasons: (i) it had already been used to approximate the DOS of the lattice vibrations by Montroll in 1942/3 [23], (ii) in the case of the frequency distribution of the normal states of a Hamiltonian describing a set of discrete atoms containing some degree of disorder, so that the cyclic boundary conditions are no more useful, physicists have found a way to calculate the moments by computing closed graphs, which one can also nicely visualize as closed walks, on the lattice. This was first found (published) by Pirenne in 1958 [24], and, independently at about the same time, by Domb, Maradudin, Montroll and Weiss in 1959 [25], for the study of vibrations on disordered systems (viz. crystals containing isotopes randomly distributed, or mixed binary alloys). In the case of Domb et al., the harmonic interactions were limited to first neighbour interactions (as it is the case in tight binding) and they could develop more applicable relations than Pirenne's general formulae. Thus this naturally was a method of choice for Fr. Cyrot-Lackmann (and Friedel) as she wanted to study the cohesion of liquid transition metals (after discussions with Loup Verlet who was working in the nearest neighbour university building in Orsay) [26]. It was also well suited to calculate surface tensions as there are simply fewer closed graphs related to surface atoms than to bulk atoms. 


\section{The Seventies (1970) and early Eighties; François Ducastelle and the second moment interaction model}

After computing the cohesive energy of a solid, the next idea naturally was to investigate its elastic properties. It was however rapidly realized that the simple rigid band model could not reproduce the bulk modulus (the lower the first nearest neighbour distance, the larger the $\beta$ overlap interaction, thus the larger the band and the larger the cohesive energy.) [27-28]. It was not possible to go further in a simple way with this band model in order to reproduce even just the main features of the experimental data collected by Gschneider Jr. for the equilibrium atomic volumes, the bulk moduli and the shear moduli which also exhibit parabolic shapes for the transition metal series, at least for the $4 d$ and $5 d$ series (Gschneider's figures 11, 6 and 3 respectively in his 1964 review paper [29]. The $3 d$ series is complicated by magnetism). François Ducastelle, Françoise Cyrot-Lackmann's thesis student, was the first to take this problem over by adding to the purely binding band contribution (purely binding in the simple model adopted) a purely repulsive contribution as a simple sum of repulsive pairwise interactions which he took of the Born-Mayer, $\exp (-p R)$, form, a form particularly suited to the exponential $R$-form (see Appendix C) of the overlapping integral $\beta$ chosen by Friedel at interatomic distances in the equilibrium condensed state (on the account that "their [small] number of spherical [nodes] allows them [the atomic $d$-orbitals] to decrease exponentially as $\exp (-q R)$ at [relatively short] distance from the origin" [30]). With respect to the usual all-pairwise potentials (e.g. Born, Lennard-Jones) it was important to clearly emphasize that the band binding contribution " $E_{\mathrm{B}}$ ne peut pas s'écrire comme une somme de contributions de paires d'atomes bien que $\mu_{2}$ s'obtienne de cette façon, ceci parce que $E_{B}$ est proportionnel à $\bigvee_{\mu_{2}}$ et non à $\mu_{2}$." [31]. With this natural and consistent choice of exponential functions, François Ducastelle could develop some relatively simple formulae for the equilibrium parameter, the total cohesive energy at equilibrium, the bulk modulus, and the cubic elastic shear moduli for various elementary atomic structures. For the fcc case for instance, he obtained $c_{44}$ and $c^{\prime}$ both proportional to 1-2q/p. François also obtained $\mathrm{c}_{44} / \mathrm{c}_{12}=(1-2 q / p) /(1-2 q / 3 p)$, and, in order to get a good agreement with experimental data, he indicated that $p$ should be comprised between $3 q$ and $5 q$. Thus, François Ducastelle could reproduce rather well, at least for the fcc transition metals, the non negligible departures from the Cauchy relationship for the cubic structures $\left(c_{12}=c_{44}\right.$, not to be confused with the $c^{\prime}=c_{44}$ isotropic relationship), a feature that a pairwise potential could absolutely not reproduce, 
whatever its parameterisation (for some comments on the Poisson-Cauchy relations, see Appendix D).

After reproducing the structural and cohesive properties of a solid at, and around its equilibrium state, the following step was to look after the properties of structural defects such as surfaces and vacancies (to start with): their energies of formation and their relaxed atomic structures. For instance it was known that the classical (non oscillatory) all-pairwise atomic potentials give a vacancy energy formation equals to the cohesive energy per atom, which contradicts experimental data for metals by a factor of about three to four, and that they predict an outward relaxation for surfaces when an inward relaxation is usually measured (much less was known about the atomic relaxations around a vacancy). The conceptual difficulty was, however, to go beyond the paradigm of periodicity, so connected to the paradigm of reciprocal space. It is easy to realize, afterwards, that the tight binding and moment approach is actually made for that. It proved difficult to realize it spontaneously at that time and this only came slowly. One had to be sure that no hypothesis or condition was violated, notably self-consistency.

In the manuscript of his thesis, Fr. Ducastelle showed that, because the number of $d$ electrons per atom in the solid does not change under a small elastic transformation, the formula $E_{\mathrm{B}}=$-const $\cdot \beta_{\mathrm{o}} \sqrt{\sum_{j} e^{-2 q R_{j}}}$ is valid for any filling of the $d$ band. In his article, however, he just wrote 'il est facile de voir' and did not even give that formula which, obviously for us now, can be re-written as $E_{\mathrm{B}}=$-const $\cdot \beta_{\mathrm{o}} e^{-q R} \sqrt{z_{c}}$ for a perfect, or only elastically deformed, crystalline structure, and to $E_{\mathrm{B}}(i)=-$ const $\cdot \beta_{\mathrm{o}} e^{-q R} \sqrt{z_{c}(i)}$ for every atom $i$ in a complex structural defect provided electrical neutrality is maintained at first order of approximation for each atom, which is true in metals thanks to metallic screening. It was simply beyond preoccupations in 1970, and even in the simple case of perfect crystal, the (already solved) problem was raised eighteen years later by Ackland, Finnis and Vitek about the Gaussian formulation [32]. The choice of a Gaussian $n_{d}(E)$, instead of the rectangular function originally used by Friedel in 1964, actually rendered the 'il est facile de voir' less straightforward than in the rectangular case where $E_{\mathrm{B}}=-\left[\left(n_{d}^{2}-10 n_{d}\right) / 20\right] \cdot|\beta| \sqrt{z_{c}}$ which obviously gives $E_{\mathrm{B}}=$-const $\cdot \beta \mid \sqrt{z_{c}}$ if $n_{d}$ remains constant per atom (see Appendix A). The choice of a Gaussian $n_{d}(E)$ came from the fact that it is the first level of the Edgeworth series which can naturally include higher order moments [22]. Within the second moment approximation, Ackland et al. [32] provided a mathematical demonstration of the validity of 
the proportionality to $\sqrt{\mu_{2}(i)}$, and thus to $\sqrt{z_{c}(i)}$, in any local environment which preserves the local charge neutrality and the original symmetry of the local DOS (only compression or stretching by a constant factor, plus global shift to maintain charge neutrality).

Considering electrical neutrality at the atomic level even around a vacancy also means neglecting the Friedel charge oscillations, which is entirely valid if one is not trying to analyse them, as we now know for sure. Might have it represented a conceptual difficulty for the students of Friedel in spite of Friedel's own pragmatism? It simply was not that obvious, and, whereas some people made it right in the details of their calculations, some people made it wrong. Sutton et al.'s article, with a first principles background, masterly settled the point in $1988[33]$.

Yet, in 1976, Allan \& Lannoo had already considered these problems, in an article which contains a discussion of the influence of self-consistency on the relaxation near a vacancy in transition metals [34]. They had also obtained a very simple formula for the fcc vacancy formation energy: $E_{\mathrm{vac}} \sim E_{\mathrm{coh}} \cdot(1-2 q / p) /(2[1-q / p])$ (to first order in $\left.1 / z_{\mathrm{c}}=1 / 12\right) . E_{\mathrm{coh}}$ is the corresponding cohesive energy, with respect to neutral free atoms), which gives a ratio $\mathrm{E}_{\mathrm{vac}} / \mathrm{E}_{\mathrm{coh}}$ comprised between 0.25 and 0.375 for $3 q \leq p \leq 5 q$, which sensibly agrees with experimental values (first nearest neighbour pair potentials give $\mathrm{E}_{\mathrm{vac}}=\mathrm{E}_{\mathrm{coh}}$ ).

Allan and Lannoo [34-36], Gordon, Cyrot-Lackmann and Desjonquères [37], Masuda and Sato [38], Gupta [39], Desjonquères and Spanjaard [40-41], Tomanek and Bennemann [42]: They all used Ducastelle's model to investigate more or less complex systems, including adatoms, small clusters, screw dislocation, carrying out some more or less extended relaxation on the atomic positions near the defect, always trying to keep closed form analytical formulae as much as possible. When it was not possible, as in the case of the screw dislocation in dilute bcc transition metal alloys, Masuda and Sato had everything to "calculate the total energy as a function of the atomic displacements" and "the atoms in the crystallite [were] relaxed along the screw dislocation." [38]

\section{1984; the Finnis-Sinclair article}

So what was new with Finnis and Sinclair 1984 [43], who acknowledged they chose " $f(\rho)$ to be $\sqrt{ }_{\rho}$, so as to mimic the result of tight-binding theory"? I see several important points (from $a$ to $f)$ :

a. The title: "A simple empirical N-body potential for transition metals." By contrast with previously mentioned articles, they do not announce the calculation of a specific property but 
they present a tool.

$b$. Their pragmatism: "the present approach is empirical", "the consequences of the form of the model (...) do not depend on the physical interpretation." One may compare this attitude with Newton's "hypotheses non fingo." Pragmatism also includes some amount of antiCartesianism.

c. A general program: "for the purpose of atomistic simulation", with explicit consideration for forces at the atomic level: "In order to perform atomistic simulations of crystals containing defects it is essential to have a rapid algorithm for calculating the force on each atom." They do provide the reader with general analytical formulae for calculation of forces. This will allow for Molecular Dynamics, not just static minimization. (On MD simulations using nbody potentials, see Appendix E.)

$d$. The Zeitgeist: Daw and Baskes had just published their two articles [44-45]. So it was in the air. In an interview in August 2000, Michele Parrinello declared that in 1983 "we invented what is called the glue potential, on which Ercolessi wrote his thesis. He was drafted for military service, and the work was stopped for a year and a half. During that time other people had similar ideas, and applied them in metals. So due to the draft we lost time, and bit of priority on this problem, but that's life." [46]

Yet there are obvious philosophical differences between these three approaches:

(i) Ercolessi, Parrinello and Tosatti declared that "although further theoretical justifications of this scheme can be found in the so-called embedded-atom method," they did "not believe that a truly first principles derivation can be given" [47] and they worked out purely empirically constructed analytical functions, carried out once, for gold [48].

(ii) Daw and Baskes used purely numerical functions deduced from first principle calculation or via a semi-empirical approach. These functions can only be tabulated and plotted.

(iii) Finnis and Sinclair used simple analytical functions, with the square root embedding function, "so as to mimic the result of tight-binding theory."

The origin of Daw and Baskes Embedded Atom Method has later been traced back, probably by Foiles [49-50], to Friedel 1952 impurity-in-jellium model [51] (see Appendix F). The basic idea from which Daw and Baskes drew on is that an atom in a metallic environment does not feel the detailed structure of the nearby atoms due to the effective screening in metallic systems. Each atom is considered as hosted in a homogeneous gas (LDA spirit) and its energy becomes a functional of the local host electron density in which it is immersed. With this approach which only considers the local electronic density, it is difficult to distinguish between fcc and hcp structures for instance. A pragmatic way to go is to consider 


\section{Acknowledgments}

I thank Professor Jacques Friedel, as well as François Ducastelle, for many invaluable discussions, by post mail and email. I also wish to thank the library department of the École Polytechnique for providing me with many ancient articles and books.

\section{Appendix A: the origins of the moments and the method of moments}

A known positive function (reasonably well behaved, e.g. a weight function, a probability distribution function) can be geometrically described (analysed) through its moments (which are usually normed and centred, but attention must be paid if a more general formalism is adopted). The zeroth order one corresponds to its surface (its mass, usually normed to 1), the first order moment gives the centre (centre of mass, the mean value of the weighted $X$-line), with the second order moment one can get a measure of the 'width' of the function (the root mean square deviation with respect to the chosen origin, directly the standard deviation if it is with respect to the centre of the function), with the third order moment combined with the 
previous one one can get a measure of the asymmetry of the function, and with the fourth order moment one can have some indication on the mono or bimodality of the function.

If one does not know the function by itself, but can have access to its first moments (one way or another), one then has methods to approximate that function although the approximation is not unique and may not be too good if one is after a precise description of the function (this is usually not the case at the beginning of a new field of research, as with Friedel in the $60 \mathrm{~s}$, but it rapidly turned out to be in the $80 \mathrm{~s}$ ). The expression of a distribution as a function of its moments does not constitute a converging series at all. When the number of moments gets large, an elegant solution to that so-called moment problem is based on the continued fraction technique. This is a mathematical problem which goes back to I.J. Bienaymé (1852) and P.L. Čebyšev who is the founder of the use of continued fractions for the method of moments (Tchebychef 1874, his disciple Markov in 1884, and Stieltjes, independently, in 1894). The solution is also closed to the recursive method (Lanczos' method, see [3-4], and all contributions by Heine, Haydock, etc., in [53]). For the historical connection between continued fractions and Padé approximants, see [54].

The 'second moment' is the second order moment, thus the third moment. As usual, beware of notations and conventions, normalisation (yes or not, per atom, or even per electronic state if they are all equivalent.) and choice of origin (e.g. centred or not).

With the following notation, $m_{n}=\int X^{n} f(X) d X ; \alpha_{n}=\frac{1}{m_{0}} \int X^{n} f(X) d X=\overline{X^{n}}$ and $\mu_{n}=\frac{1}{m_{0}} \int\left(X-\alpha_{1}\right)^{n} f(X) d X=\overline{x^{n}}$ with the centred variable $x=X$ - $\alpha_{1}$, I simply recall the following classical results (e.g. see [55]):

\begin{tabular}{|l|l|l|l|l|}
\hline$\mu_{0}=1$ & $\mu_{1}=0$ & $\mu_{2}=\overline{x^{2}}$ & $\mu_{3}=\overline{x^{3}}$ & $\mu_{\mathrm{n}}=\overline{x^{n}}$ \\
\hline$\alpha_{0}=1$ & $\alpha_{1}=\bar{X}$ & $\alpha_{2}=\mu_{2}+\alpha_{1}^{2}=\mu_{2}+\bar{X}^{2}$ & $\alpha_{3}=\mu_{3}+3 \alpha_{1} \mu_{2}+\alpha_{1}^{3}$ & $\alpha_{\mathrm{n}}$ \\
\hline $\mathrm{m}_{0}=S$ & $\mathrm{~m}_{1}=S \alpha_{1}$ & $\mathrm{~m}_{2}=S \alpha_{2}$ & $\mathrm{~m}_{3}=S \alpha_{3}$ & $\mathrm{~m}_{\mathrm{n}}=S \alpha_{\mathrm{n}}$ \\
\hline
\end{tabular}

From the notation $\mu_{2}: \sigma^{2}$, one gets $\sigma^{2}=\alpha_{2}-\alpha_{1}^{2}=\bar{X}^{2}-\bar{X}^{2}$.

When the distribution is known, the $\mu_{n}$, because they are centred, are the easiest moments to calculate.

The second moment approximation does not mean that there is a series which is truncated to its second moment (or third term). It means that no other parameter is available. 
Yet, with only three moments, the distribution can be rectangular, Gaussian, parabolic, ... For instance let us consider a rectangular distribution of surface $S$, of width $w$ and centred on $M$, and a Gaussian (Laplace-Gauss) distribution of surface $S$, of half 'width' $\sigma$ at $1 / \sqrt{ }$ e height and centred on $M$. These two distributions being symmetric with respect to their centres $\mathrm{M}$, their odd centred moments are null. Their even moments $\mu_{2 n}$ are $w^{2 n} /\left[(2 n+1) 2^{2 n}\right]$ for the rectangular distribution, and $\sigma^{2 n}(2 n) ! /\left(2^{n} n\right.$ !) for the Gaussian distribution. Thus nothing more than three parameters, $S, M$ and $w$, or $S, M$ and $\sigma$, are required to entirely describe these two distributions and their moments. Conversely, three parameters, $\mathrm{m}_{0}, \mathrm{~m}_{1}$ (or $\left.\alpha_{1}\right)$ and $\mathrm{m}_{2}$ (or $\alpha_{2}$ or $\mu_{2}$ ) can define a rectangular or a Gaussian distribution. These two distributions obviously have different shapes (and different moments beyond $\mu_{2}$, e.g. $\mu_{4}$ equals $144 \mu_{2}^{2} / 90$ for the rectangular case, and $3 \mu_{2}^{2}$ in the Gaussian case).

For an elementary crystal which can be described by a tight-binding Hamiltonian, one considers that every atom has $\mathrm{m}_{0}$ equivalent electronic state available (10 for the $d$ states, spin state included), and the $\{$ Pirenne-Domb et alii-Cyrot-Lackmann $\}$-theorem gives $\alpha_{1}=E_{0}$, the inner atomic level which is equal to the atomic level $E_{a t}$ plus a shift $\alpha$ due to the fact that the atoms are not isolated, and $\mu_{2}=z_{c} \beta^{2}$ (there are $z_{c}$ closed walks which go to a first neighbour and thus contribute, way and return, $\beta \cdot \beta$ ). Assuming a rectangular distribution, one thus gets $w^{2} / 12=\mathrm{z}_{\mathrm{c}} \beta^{2}$ hence $w=\sqrt{ }_{12} \sqrt{ }_{\mathrm{c}}|\beta|$ whereas $n_{d}\left(E_{F}\right)=\int_{E_{0}-w / 2}^{E_{\tilde{c}}} A d E=\frac{10}{w}\left[e_{F}+w / 2\right]$ (since $S=A w=\mathrm{m}_{0}=10$ and noting $e$ the centred variable $\left.e=E-E_{0}\right)$ and $E_{B}=\int_{E_{0}-w / 2}^{E_{E}}\left(E-E_{a t}\right) A d E \sim \frac{10}{2 w}\left[e_{F}^{2}-(w / 2)^{2}\right]$ if one neglects the shift $\alpha$ for the sake of clarity, which gives, after some simple algebraic manipulations, $E_{B}=-2.5 w+\frac{w}{20}\left[n_{d}\left(E_{F}\right)-5\right]^{2}$, a shifted parabola in $n_{d}\left(E_{F}\right)$, and also a linear function in $w$, hence $E_{\mathrm{B}}=$-const $\cdot|\beta| \sqrt{z_{c}}$. Assuming a Gaussian distribution does not alter the conclusions but makes the demonstration purely numerical for the parabolic shape, and more subtle (at least to my taste) for the linear dependence in $|\beta| \sqrt{z_{c}}$. In his truly remarkable sometimes intuitive way, Friedel 'knew' in 1964 that these results would not depend much on the shape of $n_{d}(E)$. Yet, as far as I am concerned, I can understand it only through the demonstrative work carried out by Fr. CyrotLackmann. 


\section{Note on the bimodality indicator}

Jean-Pierre Gaspard [3-4] noticed that the ratio $\tilde{b}=\left(\mu_{4} \mu_{2}-\mu_{3}^{2}-\mu_{2}^{3}\right) / \mu_{2}^{3}$, which can be deduced from the first two terms of the continued fraction of the resolvant of the distribution function, gives some indication on the mono or bimodality of a distribution. A distribution with $\tilde{b}>>1$ is monomodal whereas a distribution with $\tilde{b}<<1$ is bimodal. It is tempting to propose a critical value for $\tilde{b}$. However such a critical value does not exist in a universal way. Just to give two examples, it is 0.8 for the symmetrical beta law $\left([x(1-x)]^{a-1}\right.$, for $0 \leq x \leq 1, a$ being a real number $>0$ ) but for the symmetrical mixture of two equivalent Gaussians differing only by their centres, it is 1.5 . The case of a non symmetrical distribution will be more difficult to decide. Let us also note that, using Pearson's coefficients $\beta_{1}=\mu_{3}^{2} / \mu_{2}^{3}$ and $\beta_{2}=\mu_{4} / \mu_{2}^{2}$, one has $\tilde{b}=\beta_{2}-\beta_{1}-1$.

\section{Some original quotations which introduced the moments:}

Marin Mersenne (French monk and universal scientist, well known for being in touch with almost all savants of his time), in his free translation/adaptation in 1634 of the not yet published lectures of Galileo Galilei on Mechanics at the University of Padua in the 1590s: “Le moment eft l'inclination du mefme corps, lors qu'elle n'eft pas feulement confidérée dans ledit corps, mais conioinctement auec la fituation qu'il a fur le bras d'vn leuier, ou d'vne balance." [56]

Galileo Galilei, in Le Mecaniche, posthumously published in Ravenna in 1649: "Momento è la propensione di andare al basso, cagionata non tanto dalla gravità del mobile, quanto dalla disposizione che abbino tra di loro diversi corpi gravi" [57]

Irénée-Jules Bienaymé (1852): “(...) si l'on appelle $\mu_{\Sigma}$ la moyenne des puissances $\Sigma$ de toutes les erreurs possibles, on aura $\int \varepsilon^{\Sigma} d \varepsilon \varphi(\varepsilon)=\mu_{\Sigma}{ }^{\prime}[58]$

Pafnouty Lvovitch Tchebychev (1874): "En cherchant à tirer tout le parti possible sur les valeurs limites de l'intégrale $\int_{a}^{h} f(x) d x$ des valeurs des intégrales $\int_{A}^{R} f(x) d x, \int_{A}^{R} x f(x) d x$, $\int_{A}^{B} x^{2} f(x) d x, \int_{A}^{B} x^{m} f(x) d x$, où l'on a $A<a, B>b$, et où $f(x)$ reste positive, je suis parvenu à 


\section{Appendix B: on Tight Binding and LCAO}

Felix Bloch demonstrated (in much more details than some forerunners as Houston, Strutt, Frenkel, and quite independently) that if the ionised atoms are positioned regularly, as in a crystal, then, for the electrons in such a periodic lattice, there are solutions of Schrödinger's equation in the form of plane waves that describe electrons travelling in any direction through the crystal without being scattered [61]. The Bloch plane-wave solutions are more complicated than the de Broglie wave of an electron in free space, but a wave-vector $\boldsymbol{k}$ may still be defined: one has to deal with de Broglie waves, "die im Rhytmus des Gitteraufbaus moduliert sind." Exact solutions for any particular choice of the periodic potential $V(\boldsymbol{r})$ (: the interaction potential between the conduction electrons and the lattice) are probably impossible to find and Bloch worked out exact solutions in a limit case he knew from his time in Zürich where he, and Heitler and London enjoyed walking together. F. Bloch drew on the idea that Heitler and London had used on their treatment of the hydrogen molecule, that of constructing electron wave functions starting from a basis of unperturbed single-atom ground-state orbitals. F. Bloch called it the limit case of stark gebundener Elektronen (strongly bound electrons), which came to be know as tight-binding approximation. In his 1931 article, Erich Hückel extensively referenced F. Bloch [62]. At the request of their common director Heisenberg, Peierls, among many other things, worked out the solution for a nearly free version of $V(\boldsymbol{r})$, instead of the tight-binding version chosen by Bloch, and was delighted to get 
some similar results which were thus likely to be independent on the details of the interactions of the electrons with the atoms. A.H. Wilson developed the band theory further (see [63-65]).

Wigner and his student Seitz blurred the landscape in 1933 when they explicitly considered the work of Bloch as mainly based on the hypothesis of free electrons in chemistry [9]. Mott and Jones coined the English expression 'tight binding' approximation in their 1936 book [12]. Friedel and his co-workers always quoted Hückel for the tight-binding approximation.

Yet, in 1931, referring to F. Bloch 1928, Erich Hückel wrote: "Es lassen sich dann die Eigenfunktionen eines Elektrons durch Linearkombinationen der Eigenfunktionen des Elektrons bei getrennten Atomen annähern.” (p. 219 of [62]). And, in 1955, Reitz clearly wrote: "the linear combination of atomic orbitals, or Bloch, or tight binding method. This technique was first proposed for solids by Bloch.” (page 16 of [66])

It must be recognized that Bloch's name, because of Bloch's theorem, conveys the idea of periodicity, and Fourier transforms. By contrast, because Hückel dealt with finite size molecules, his name conveys the idea of as distorted as you like configurations with a real space approach (even if Hückel happened to also use Bloch's periodicity trick because he actually dealt with benzene, a cyclic 'aromatic' molecule!). It is also probably difficult to comprehend that some electrons may be delocalized without being totally free. The most relevant picture here is that of itinerant electrons, hopping from one atom to the next, but not necessarily forming a homogeneous jellium between the atoms as free electrons do.

Lennard-Jones is usually given as the founder of the LCAO method in 1929 [67] although this was the way of doing at that time: Hund 1927 onward, Mulliken 1928, LennardJones 1929. Mulliken formalized the technique and introduced the English term 'LCAO' in $1935[68]$.

There is a difference in spirit between Heitler and London 1927 and the so-called HundMulliken approach, at least in their premises. Heitler \& London 1927 start with atomic orbitals, AOs, (e.g. $\varphi_{\mathrm{A}}(\boldsymbol{r})$ and $\varphi_{\mathrm{B}}(\boldsymbol{r})$ ) which they combine, through multiplications and additions, to generate symmetrical and antisymmetrical functions. The building blocks of the Hund-Mulliken approach are LCAOs, sums and differences of atomic orbitals (e.g. $\varphi_{\mathrm{A}}(\boldsymbol{r})+\varphi_{\mathrm{B}}(\boldsymbol{r})$ and $\left.\varphi_{\mathrm{A}}(\boldsymbol{r})-\varphi_{\mathrm{B}}(\boldsymbol{r})\right)$ which are then combined to generate symmetrical and antisymmetrical functions. These building blocks are more appropriate when the atoms are relatively close together, yet still retaining some of their atomic character: this is the spirit of the 'tight binding' approach. 
Tight binding has also been successfully developed for covalent materials by Friedel, Leman, Lannoo, Allan, Gaspard, and others with the use of moments. We are dealing here with $s$ and $p$ electronic states, which are quite different in nature from the atomic like $d$ states. But because of the $s p^{3}$ hybridization, these materials crystallize in the cubic diamond atomic structure, which is an open structure so that the influence of the second neighbours can be neglected in a first approximation with respect to the influence of the first neighbours. A tight binding treatment is relevant, and successful. The electrons are nevertheless free to move from one atom to any other through the covalent bounds and the $E(k)$ dispersion for silicon is very close to the free electron dispersion at small $k$. The cubic diamond materials have been the first for which n-body potentials have been developed [69], although for a completely different reason. The tetrahedral angles between the $s p^{3}$ bonds is a fundamental of the cubic diamond structure and can only be reproduced with three body terms with explicit angular dependence (and it takes three points to define an angle). The Cauchy relations are violated for cubic diamond materials for two reasons: the interactions are not pairwise and the atoms are not centres of symmetry. The Cauchy discrepancy being negative, an embedded function of the usual type cannot be expected to work. The presence of explicit angular dependent terms seems necessary.

\section{Appendix C: exponential or power laws?}

The exponential form of the overlap, or transfer or hopping or bond, integral, has been chosen historically, in part because of the widespread use of the "Slater" orbitals [70]. Ab initio calculations that the dependence should rather be of the inverse power form [71-72].

In the immediate vicinity of the equilibrium first neighbour distance $R_{0}$, it does not matter since one has $\mathrm{e}^{-\kappa R} \sim c R^{-n}$ with $n=\kappa R_{o}$ and $c=\left(R_{o} / \mathrm{e}\right)^{n}$. If one wants to stretch the bonds further, i.e. to extend the validity of the overlap law, both solutions fail and pragmatism must prevail, especially for the smoothing of the function down to zero (e.g. see $\$ 7.3 .2$ of $[8])$. 


\section{Appendix D: on the Cauchy relations}

Following the mechanical school of Navier, Poisson, Cauchy, Clausius and Saint-Venant, one should have special relations between elastic constants which A.E.H. Love called Cauchy relations in the historical introduction of his famous treatise on elasticity more than a century ago [73]. These relations are consequences of the hypothesis of interactions between atomic point centres, together with other hypotheses such as pairwise interactions, atoms being symmetry centres of the structure, and no constraints at zero deformation. They proved to be incompatible with experimental measurements (once properly made..., for instance by W. Voigt) so that the atomic hypothesis was contested by positivist anti atomists such as Pierre Duhem, by no means an obscurantist. He was right in the sense that the real atoms are not point centres with no internal structure. From an opposite point of view, in the sixth volume of the Handbuch der Physik series in 1928, J.W. Geckeler wrote that for the elementary metals which violate the Cauchy relations, one must conclude that their atomic structure is not just a simple (Bravais) lattice. Still later on, Raman and his disciples in India, and Laval in France were to oppose Born about the number of independent elastic constants for diamond.

Cauchy's name is also associated to the opposite mechanical school, viz. the continuous mechanics tradition. The appellation Poisson-Cauchy relations would be more fitted, from an historical point of view.

Daw and Baskes noted that the positive curvature of the embedding function implies positive Cauchy discrepancies. Finnis and Sinclair reproduced the same remark, noting that it is the case for the square root function. Thus for metals with negative discrepancies, such as rhodium and iridium, it means that these metals have some basic properties which are not taken into account by the EAM model, for instance explicit angular dependant terms (hence the MEAMs: modified EAMs).

DFT ab initio calculations, in full electrons or with pseudopotentials, only started in the eighties, with sometimes only a moderate success as far as the calculation of elastic constants is concerned (see [1]). 


\section{Appendix E: the first MD simulations with n-body potentials}

As far as I know, the first MD simulation with a metallic n-body potential was carried out by S.M. Foiles in 1985 with an EAM potential for liquid transition and noble fcc metals (Ni, Pd, $\mathrm{Pt}, \mathrm{Cu}, \mathrm{Ag}$, and $\mathrm{Au})$ [74].

It is worth noting that at the same time, Stillinger and Weber designed an n-body potential for silicon, with explicit angular dependence, based on experimental and ab initio data, and also performed Molecular Dynamics calculations to check the liquid state [75].

Ercolessi, Parrinello and Tosatti in 1988 (published) performed MD simulations with their glue gold model in order to check the melting temperature of their model [52].

Rosato, Guillope and Legrand in 1989 are the first who carried out MD simulations with FS-like potentials, with Ducastelle's exponential functions, on fcc transition metals [76].

For sake of completeness, I also mention the MD simulations of systems of rigid molecules: When atoms of rigid molecules interact together through relatively long ranged Van der Waals interactions, one can say that the molecules, which are the elementary (rigid) bodies of the system, interact together through n-body potentials. The first MD simulation of this kind is by Barojas, Levesque and Quentrec in 1973 [77]. The interaction model was described in 1967 by Sweet and Steele [78].

For a short introduction to atomistic simulations, Monte Carlo Metropolis and Molecular Dynamics, with some historical information, see [79].

\section{Appendix F: on the origins of the EAM}

Stephen Foiles wrote in 1996 ([50], + see [49]) that "the idea of an embedding energy was first suggested by Friedel [51] and later developed further by Nørskov and Lang [effective medium theory 1980] and by Stott and Zaremba [quasi-atom concept 1980].”

In the late $40 \mathrm{~s}$, J. Friedel carried out the first numerical study of grain boundaries at the atomistic level (see [80]). Yet, a little disappointed by the crudeness of the pairwise interatomic potentials he had had to use, he decided to learn more about electronic interactions in metals and was introduced by his cousin Ch. Crussard to N.F. Mott in Bristol. There he also met Charles Frank, and his future wife.

In 1936, Mott, concerned with dilute solid solutions (metallic alloys, Hume-Rothery 
electron concentration rule (the famous $21 / 13$ ratio, in 1926), influence on electric resistivity) had considered the problem of local screening of charges around (highly dispersed) impurities in a metallic matrix, notably when the dissolved and solvent metals do not occupy the same column in the periodic table, e.g. when an atom of zinc or aluminium is dissolved in copper. "The extra electrons of the dissolved atom must be regarded as belonging to the lattice rather than to the atom. We should therefore expect the dissolved atom to carry an extra positive charge $z$, equal to 1 for zinc, 2 for aluminium" [81]. Friedel ([51, 82]) modified Mott's rapidly decaying solution (à la Debye-Hückel) into a less rapidly decaying solution with oscillations which later came to be known as 'Friedel oscillations'.

The conceptual difficulty master was to consider the extra electrons of the impurity as given to the lattice, thus unbound, and yet to have the jellium respond as a local charge concentration (electron polarization cloud). One indeed finds in Friedel 1952 the idea that "an impurity atom is embedded in a regular lattice", and that the perturbation is only local (then, self consistently, that embedded atom can only depend on its local environment).

It must however be recognized that the lack of simple guidelines, except complex ab initio calculations or optimization fitting on empirical data, is not in the spirit of J. Friedel's approach.

\section{Appendix G: unabridged references}

[1] O.B.M. Hardouin Duparc, Copper and Venus. How copper atoms interact, in Copper, edited by J.M. Welter (Wiley-VCH, Weinheim, 2006) pp.18-24.

[2] G. Allan, Elementary electronic structures in semiconductors and transition metals, in Electronic structure of crystal defects and of disordered systems, Summer School Aussois Sept. 1980, edited by Fr. Gautier, M. Gerl and P. Guyot (Éditions de Physique, Les Ulis, 1981) pp.3-44.

[3] J.-P. Gaspard, Atomic and Electronic Structures of Amorphous Systems, in Electronic Structure of Crystal Defects and of Disordered Systems, Aussois 1980, edited by Fr. Gautier, M. Gerl and P. Guyot (Éditions de Physique, Les Ulis, 1986) pp.279-317.

[4] J.-P. Gaspard, Du cristal à l'amorphe : structure et cohésion, in Structures et Instabilités, Beg-Rohu 1985, edited by C. Godrèche (Éditions de Physique, Les Ulis, 1986) pp.441-544. 
[5] F. Ducastelle, Order and Phase Stability in Alloys (North-Holland, Amsterdam, 1991).

[6] A. Sutton, Electronic Structure of Materials (Clarendon Press, Oxford, 1993).

[7] D. Pettifor, Bonding and Structures of Molecules and Solids (Clarendon Press, Oxford, 1995).

[8] M. Finnis, Interatomic forces in Condensed Matter (Oxford Univ. Press, Oxford, 2003).

[9] E. Wigner and F. Seitz, On the Constitution of Metallic Sodium, Phys. Rev. 43 (1933) pp.804-810.

[10] N.F. Mott, A Discussion of the Transition Metals on the Basis of Quantum Mechanics. Proc. Phys. Soc. 47 (1935) pp.571-588.

[11] H. Fröhlich, Elektronentheorie der Metalle (J. Springer Verlag, Berlin, 1936).

[12] N.F. Mott and H. Jones, The Theory of the Properties of Metals and Alloys (Clarendon Press, Oxford, 1936).

[13] F. Seitz and R.P. Johnson, Modern Theory of Solids, J. Applied Physics 8 (1937) pp.84$97+$ pp.186-199.

[14] E. Wigner and F. Seitz, Qualitative Analysis of the Cohesion in Metals, in Solid State Physics, edited by F. Seitz and D. Turnbull, 1 (1955) pp.97-126.

[15] J.C. Slater, The ferromagnetism of nickel, Phys. Rev. 49 (1936) pp.537-545.

[16] J.C. Slater, Wave Functions in a Periodic Potential, Phys. Rev. 51 (1937) pp.846-851.

[17] J.C. Philips, Band Theory of Transition Metals, in Theory of Magnetism in Transition Metals, Proc. 1966 Varenna (Enrico Fermi) Summer School, Course 37, edited by W. Marshall (Academic Press, New York, 1967) pp.22-49.

[18] J. Friedel, Ferromagnetic Transition Alloys, in Theory of Magnetism in Transition Metals, Proc. 1966 Varenna (Enrico Fermi) Summer School, Course 37, edited by W. Marshall (Academic Press, New York, 1967) pp.283-318.

[19] N.F. Mott, Récents progrès et difficultés de la théorie électronique des métaux, in Ferromagnétisme, volume II of Le magnétisme, International Conference on Magnetism, Strasbourg, May 1939 (CNRS-IICI, Paris, 1940) pp.1-39 (with discussions).

[20] L. Néel, Champs moléculaires, aimantation à saturation et constantes de Curie des éléments de transition et de leurs alliages, in Ferromagnétisme, volume II of Le magnétisme, International Conference on Magnetism, Strasbourg, May 1939 (CNRS-IICI, Paris, 1940) pp.65-164 (with discussions).

[21] J. Friedel, On the Possible Impact of Quantum Mechanics on Physical Metallurgy, Transactions of the Metallurgical Society of AIME 230 (1964) pp.616-632. 
[22] F. Cyrot-Lackmann, Sur le calcul de la cohésion et de la tension superficielle des métaux par une méthode de liaisons fortes, J. Phys. Chem. Solids 29 (1968) pp.1235-1243.

[23] E.W. Montroll, Frequency Spectrum of Crystalline Solids, Journal of Chemical Physics 10 (1942) pp.218-229, 11 (1943) pp.481-495.

[24] Jean Pirenne, Dynamics of a crystal lattice containing isotopes, Physica 24 (1958) pp.7392.

[25] C. Domb, A.A. Maradudin, E.W. Montroll and G.H. Weiss, Vibration Frequency Spectra of Disordered Lattices. I. Moments of the Spectra for Disordered Linear Chains, Phys. Rev. 115 (1959) pp.18-24.

[26] F. Cyrot-Lackmann, On the Electronic structure of Liquid Transitional Metals, Advances in Physics 16 (1967) pp.393-400.

[27] S. Barisic, J. Labbé and F. Cyrot-Lakmann, Anomalies élastiques associées aux singularités de Van Hove de la densité d'états électronique d'une bande $s$ étroite, Journal de physique 30 (1969) pp.955-965.

[28] F. Ducastelle, personal communication, 2009.

[29] K.A. Gschneider Jr., Physical Properties and Interrelationships of Metallic and Semimetallic Elements, in Solid State Physics, edited by F. Seitz and D. Turnbull, 16 (1964) pp. $275-426$.

[30] J. Friedel, Transition metals. Electronic structure of the d-band. Its role in the crystalline and magnetic structures, in The Physics of Metals. 1. Electrons, edited by J.M. Ziman as a tribute to Sir N.F. Mott (Cambridge Univ. Press, Cambridge, 1969) pp.340-408.

[31] F. Ducastelle, Modules élastiques des métaux de transition, Journal de Physique 31 (1970) pp.1055-1062.

[32] G.J. Ackland, M.W. Finnis and V. Vitek, Validity of the second moment tight-binding model, J. Phys. F 18 (1988) pp.L153-L157.

[33] A.P. Sutton, M.W. Finnis, D.G. Pettifor and Y. Ohta, The tight-binding bond model, J. Phys. C 21 (1988) pp.35-66.

[34] G. Allan and M. Lannoo, Vacancies in Transition Metals. Formation energy and Formation volume, J. Phys. Chem. Solids 37 (1976) pp.699-709.

[35] G. Allan and M. Lannoo, Relaxation near Transition Metal Surfaces, Surf. Sci. 40 (1973) pp.375-387.

[36] G. Allan and M. Lannoo, Influence of Self-Consistency on the Relaxation near a Vacancy or a Surface in Transition Metals, Phys. Status Solidi (b) 74 (1976) pp.409-414. 
[37] M.B. Gordon, F. Cyrot-Lackmann and M.C. Desjonquères, Relaxation and stability of small transition metal particles, Surf. Sci. 80 (1979) pp.159-164.

[38] K. Masuda and A. Sato, Electronic theory for screw dislocation motion in dilute b.c.c. transition metal alloys, Phil. Mag. A 44 (1981) pp.799-814.

[39] R.J. Gupta, Lattice relaxation at a metal surface, Phys. Rev. B 23 (1981) pp.6265-6270.

[40] M.C. Desjonquères and D. Spanjaard, A simple chemisorption theory and its application to transition adatoms on transition metals, J. Phys. C 15 (1982) pp.4007-4021.

[41] D. Spanjaard and M.C. Desjonquères, Comment on "Universal features of bonding in metals", Phys. Rev. B 30 (1984) pp.4822-4827.

[42] D. Tomanek and K.H. Bennemann, simple theory for the electronic and atomic structure of small clusters, Phys. Rev. B 28 (1983) pp.665-673. (+ erratum 1984).

[43] M. Finnis and J.F. Sinclair, A simple empirical N-body potential for transition metals, Phil. Mag. A 50 (1984) pp.45-55.

[44] M.S. Daw and M.I. Baskes, Semiempirical, Quantum Mechanical Calculation of Hydrogen Embrittlement in Metals, P.R.L. 50 (1983) pp.1285-1289.

[45] M.S. Daw and M.I. Baskes, Embedded-atom method: Derivation and application to impurities, surfaces, and other defects in metals, Phys. Rev. B 29 (1984) pp.6443-6453.

[46] M. Parrinello, Interview by Dónal Mac Kernan, Simu-Newsletter 2 (2000) pp.5-14.

[47] F. Ercolessi, E. Tosatti, M. Parrinello, Au(100) Surface Reconstruction, P.R.L. 57 (1986) pp. 719-722.

[48] F. Ercolessi, M. Parrinello, E. Tosatti, Simulation of Gold in the glue model, Phil. Mag. A 58 (1988) pp. 213-226.

[49] M.S. Daw, S.M. Foiles, M.I. Baskes, The embedded-atom method: a review of theory and applications, Mater. Sci. Rep. 9 (1993) pp.251-310.

[50] S.M. Foiles, Embedded-Atom and Related Methods for Modeling Metallic Systems, MRS Bulletin 21\#2 (1996) pp. 24-28.

[51] J. Friedel, The Distribution of Electrons Round Impurities in Monovalent Metals, Phil. Mag. 43 (1952) pp.153-189.

[52] G.J. Ackland, G. Tichy, V. Vitek and M.W. Finnis, Simple N-body potentials for the noble metals and nickel, Phil. Mag. A 56 (1987) 735-756.

[53] The Recursion Method and its Applications, Imperial College Sept. 1984, Proceedings edited by D.G. Pettifor and D.L. Weaire (Springer, Berlin, 1985).

[54] Cl. Brezinski, The long History of Continued Fractions and Padé Approximants (Springer, Berlin, 1991). 
[55] G. Udny Yule, An Introduction to the Theory of Statistics (Ch. Griffin, London 1911, + enlarged and revised versions with M.G. Kendall).

[56] M. Mersenne, Les Mechaniques de Galilée Florentin, Ingenieur et Mathematicien du Duc de Florence, traduites de l'italien par le Père Marin Mersenne, Minime, avec plusieurs additions de lui-même (Henry Guenon, Paris, 1634).

[57] G. Galilei, Le Mechaniche (Ravenna 1649), for instance in Le opere di Galileo Galilei, vol. 2, (Ediz. Nazionale, Florence, 1891) pp. 147-191.

[58] I.J. Bienaymé, Mémoire sur les probabilités des erreurs d'après la méthode des moindres carrés, J. Mathématiques Pures et Appliquées, Ière série 17 (1852) pp.33-78.

[59] P. Tchebychef, Sur les valeurs limites des intégrales, Journal des mathématiques pures et appliquées 19 (1874) pp.157-160.

[60] Th.-J. Stieltjes, Recherches sur les fractions continues, Annales de la Faculté des Sciences de l'Université de Toulouse pour les sciences mathématiques et physiques, $1^{\text {ère }}$ série, 8 (1894) pp.1-122 (+ (1895) pp.1-64).

[61] F. Bloch, Über die Quantenmechanik der Elektronen in Kristallgittern, Zeitschrift für Physik 52 (1928) pp.555-600.

[62] E. Hückel, Quantentheoretische Beiträge zum Benzolproblem, Zeitschrift für Physik 70 (1931) pp.204-286.

[63] Sir Nevill Mott et al., The Beginnings of Solid State Physics, Proc. Royal Society London A 371 (1980) pp.1-177.

[64] L. Hoddeson, G. Baym and M. Eckert, The development of the Quantum Mechanical Electron Theory of Metals, 1926-1933, in Out of the Crystal Maze, edited by L. Hoddeson, E. Braun and S. Weart (Oxford Univ. Press, Oxford, 1992) pp.88-111.

[65] Sir Brian Pippard, Electron in Solids, in Twentieth Century Physics, edited by L.M. Brown, A. Pais and Sir Brian Pippard, vol. III (IOP, Bristol, 1995) pp.1279-1383.

[66] J.R. Reitz, Methods of the One-Electron Theory of Solids, in Solid State Physics, edited by F. Seitz and D. Turnbull, 1 (1955) pp.1-95.

[67] J.E. Lennard-Jones, The Electronic Structure of Some Diatomic Molecules, Trans. Faraday Soc. 25 (1929) pp.668-686.

[68] R.S. Mulliken, in Electronic Structures of Polyatomic Molecules and Valence. VI. On the Method of Molecular Orbitals, J. Chem. Phys. 3 (1935) pp.375-378.

[69] P.N. Keating, Effect of Invariance Requirements on the Elastic Strain Energy of Crystals with Applications to Diamonds, Phys. Rev. 145 (1966) pp.637-645.

[70] J.C. Slater, Atomic shielding constants, Phys. Rev. 36 (1930) pp.57-64. 
[71] V. Heine, s-d Interactions in Transition Metals, Phys. Rev. 153 (1967) pp. 673-682.

[72] W.A. Harrison, Electronic Structure and the Properties of Solids, (W.H. Freeman and Co., San Francisco, 1980).

[73] A.E.H. Love, A treatise on the mathematical theory of elasticity, (Univ. Press, Cambridge, 1892 1st edition).

[74] S.M. Foiles, Application of the embedded-atom method to liquid transition metals, Phys. Rev. B 32 (1985) pp.3409-3415.

[75] F.H. Stillinger and Th.A. Weber, Computer simulation of local order in condensed phases of silicon, Phys. Rev. B 31 (1985) pp.5262-5271.

[76] V. Rosato, M. Guillope and B. Legrand, Thermodynamical and structural properties of f.c.c. transition metals using a simple tight-binding model, Phil. Mag. A 59 (1989) pp.321336.

[77] J. Barojas, D. Levesque and B. Quentrec, Simulations of Diatomic Homonuclear Liquids, Phys. Rev. A 7 (1973) pp.1092-1104.

[78] J.R. Sweet and W.A. Steele, Statistical Mechanics of Linear Molecules. I. Potential Energy Functions, J. Chem. Phys. 47 (1967) pp.3022-3028.

[79] O.B.M. Hardouin Duparc, Atomistic Simulation, in Computer Simulation in Materials Science, NATO ASI Oléron 2005, edited by H. Kirchner, L. Kubin and V. Pontikis, (Kluwer, Dordrecht, 1996) pp.63-83.

[80] O.B.M. Hardouin Duparc, Charles Crussard's early contributions: Recrystallization in situ and a Grain Boundary study with J. Friedel and B. Cullity, Int. J. Mat. Res. 10 (2009) to be published.

[81] N.F. Mott, The Electrical Resistance of Dilute Solid Solutions, Proc. Camb. Phil. Soc. 32 (1936) pp.281-290.

[82] J. Friedel, Electronic Structure of Primary Solid Solutions in Metals, Advances in Physics 3 (1954) pp.446-507. 


\section{On the origins of the Finnis-Sinclair potentials}

Olivier Hardouin Duparc

LSI, CEA-X-CNRS, UMR 7642, École Polytechnique, 91128 Palaiseau Cedex, France

\section{Contents:}

\section{Introduction}

2. The Thirties; first steps, nullified by sensible hesitations

3. The Sixties; the time of Jacques Friedel and his $d$ band model

4. The Seventies (1970) and early Eighties; François Ducastelle and his followers

5. 1984; the Finnis-Sinclair article

6. Short conclusion; for a continuing story

Acknowledgments

Appendix A: the origins of the moments and the method of moments

Appendix B: on Tight Binding and LCAO

Appendix C: exponential or power laws?

Appendix D: on the Cauchy relations

Appendix E: the first MD simulations with n-body potentials

Appendix F: on the origins of the EAM

Appendix G: unabridged references 
I trace back the origins of the famous Finnis-Sinclair potentials. These potentials mimic the results of tight binding theory through their use of the square root embedding function. From the tentative beginnings of tight binding in the 1930s up to 1984 or so, some of the famous names involved are Bloch, Seitz, Montroll, Friedel, Cyrot-Lackmann, Ducastelle, to name just a few. The application of the method of moments to the description of densities of states and its connexion to the physics of closed paths linking nearest neighbours interacting atoms helped to formalize Friedel's rectangular band model for the $d$ electrons in transition metals. Extension from perfectly periodic structures to defective ones could not be but a slow process due to the change of paradigm for solid state scientists and to the necessary caution to be paid to self-consistency. The British scientists school also contributed significantly in the $80 \mathrm{~s}$. Computer progress and pragmatism helped to go from mainly analytical developments to numerical experiments (another change of paradigm). I also digress on various not so well known historical points of interest to this story.

Keywords: Finnis-Sinclair Potentials; tight binding; LCAO; method of moments; second moment approximation; transition metals; semiconductors; Cauchy relations; embedded atom method; molecular dynamics; history of science

\section{Introduction}

Without going back to ancient Greek models (see [1]), one can say that pair potentials proved to be surprisingly efficient, at least as a first approximation, for metals and ionic crystals since Mie (1903), Grüneisen (1911-1912), Born (with Landé 1918, with Mayer 1932). Yet the consideration that metals are mainly governed by electrons moving more or less freely (Riecke, Drude, Lorentz, Thomson [Clausius-Boltzmann gas], Sommerfeld [Fermi gas] 1928) as in a gigantic molecule which should be treated through the Schrödinger equation (not to mention Dirac's equation) rapidly raised questions. Solving the Schrödinger equation for a large number of atoms in a condensed matter state, however, implies a lot of numerical work (now computer work) which restricts the size of the systems which can be considered. Most physicists have always tried to use, as much as possible, simple models endorsed with some physically interpretable parameters which can be varied at will (for numerical experimentation) and/or fitted versus trustable data (experimental and/or ab initio, when reliability is warranted) for real materials. Simplicity, for understanding, versus realism,

\footnotetext{
*Corresponding author. Email: olivier.hardouinduparc@polytechnique.edu
} 
depending on the (complexity of the) question to be addressed: This depends on the conceptual tools, together with the numerical tools, that the physicist may use and master (e.g. irrational numbers and logarithms on one side, and computers on the other side). Conceptual tools for understanding evolve with numerical tools (and technology), and they also evolve in the sense that they may seem odd at first sight and slowly become natural once one gets used to them. They subsequently can be improved. It is this slow evolutionary process that I see at work in what led to what are now called the "Finnis-Sinclair potentials." My plan will simply follow history, starting in the 30s up to the 80s. I put in appendices some lateral information which I believe is important and not too well-known. I otherwise assume that the reader is acquainted with classical texts such as [2-8].

\section{The Thirties; first steps, nullified by sensible hesitations}

In 1935, naturally impressed by the recent breakthrough of Wigner and Seitz (1933, [9]) who had been able to account "quantitatively for the cohesive forces in a typical metal (sodium)," Mott attempted to "give a qualitative discussion, based on the same theory, of some properties of the transition metals" and in particular of the elements nickel and palladium. He considered the outermost $s$ electrons as responsible for the cohesion (and the $d$ electrons as responsible for the magnetic properties) and he estimated to 0.6 the number of $s$ electrons per atom for nickel. In a clever way, he concluded that nickel must have a binding energy greater than that of copper, which agrees with experimental data. Apart from nickel, palladium and platinum, the binding energy of transition metals was not considered and the rest of his article was mainly concerned with the discussion of magnetic susceptibilities and electrical conductivities $[10]$.

In 1936, H. Fröhlich endorsed this analysis in his textbook for all transition metals in a forcedly odd way ( $\$ 30$ of [11]) while Mott and Jones wrote that as far as transition metals are concerned, "no calculations of the binding forces in these metals have yet been made" (sic, Chapt. IV, § 3.3 of [12]).

In 1937, in the second of a set of three highly pedagogical articles, Seitz and Johnson proposed that "as long as the electrons fill depressed zones [the lower states of the bands] we may expect the binding to increase, but when levels which are higher than the atomic states are filled, we may expect the binding to decrease. That is, we expect the binding energy in one of the long periods $[3 d, 4 d, 5 d]$ to increase with atomic number at first, reach a maximum and then decrease again." [13]. This simple tight-binding picture naturally implied a number 
of approximations they perfectly knew about and explained in their series of articles. Seitz only briefly alluded to this scheme in his 1940 book but he re-expressed it, more clearly, in 1955 with Wigner in the first volume of the Seitz and Turnbull's Solid State Physics series, stating that in the case of separated $p$ or $d$ bands, "the bands are centered essentially at the value of the atomic level as long as the wave functions are assumed to be composed of atomic functions in the manner [of the tight binding approximation]. The binding is largest for the first electron in each shell in this approximation, is zero for the electron in the middle of the shell, and is negative for the electrons in the second half of the shell. This means that the cohesive energy should attain its maximum near the center of the shell and decrease toward either end." And they provided the reader with a figure of the experimental cohesive energy of the condensed state as a function of the atomic number [14]. This seems to have gone rather unnoticed.

Also in the US, however (and even in the same State of New York), Slater wrote in 1936 that the electrons in the incomplete atomic $d$ shells can be responsible for magnetism but "do not take part in the cohesion," "cannot be engaged in cohesion" [15]. He also wrote that "Overlapping the band of $3 d$ electrons is another band, arising from the $4 s$ level of the atom, with interactions with the $4 p$ and other higher levels. (...) Electrons in this band act like free electrons (...) they contribute strongly to the cohesion." Slater was thus threatening the principle of LCAO calculations that neglected excited atomic states with higher energies but larger overlap and he developed in 1937 a model where the trial wave functions are expanded in terms of plane waves within spheres centred on the nuclei [16]. This celebrated muffin-tin model involves complex calculations the results of which were not easy to interpret (see [17$18])$.

At the end of the thirties, just before the Second World War broke out, discussions about magnetism at an international conference in Strasbourg simply questioned the possibility that $d$ electrons could form bands at all [19-20].

\section{The Sixties; the time of Jacques Friedel and his $d$ band model}

After the War, matters settled down, and in 1964, in his Institute of Metals Lecture, J. Friedel independently re-developed a tight-binding analysis of transition metals concentrating on the $d$ band only, in a simpler and more precise way [21]. In this Lecture, Friedel introduced his famous rectangular $d$ band model: "For all reasonable forms $n_{d}(E)$ of the $d$ band, one finds much the same parabolic variation as when $n_{d}=$ const." He then provided the reader with an 
equation which does give the parabolic variation of the atomic cohesive energy (with respect to isolated, free atoms, the sublimation energy) with the number of $d$ electrons per atom. (The direct proportionality to the electronic hopping integral between first neighbours $\beta$ was also clear but the proportionality to the square root of the coordination number of the structure, obviously a secondary point at that time, was missed in the equation simply because the width of the $d$ band was taken as $2|\beta|$, instead of $\sqrt{12}_{12} \sqrt{\mathrm{c}}_{\mathrm{c}}|\beta|$ as it should be for the rectangular $d$-band where $\mathrm{z}_{\mathrm{c}}$ is the coordination number: the number of nearest neighbours per atom. Note that for the real $d$-band, the width is of the order of $2 z_{c}|\beta|$. For the Gaussian $d$-band, the width (support) is infinite and one defines instead the standard deviation $\sigma=\sqrt{ }$ var $=\sqrt{ } \mu_{2}$ which equals $V_{Z_{c}}|\beta|$. See Appendix A).

Françoise Cyrot, née Lackmann, substantiated her thesis director's assertion about "all reasonable forms $n_{d}(E)$ " in her $\mathrm{PhD}$ thesis (and in [22], in French). For that, she developed the integrals $n_{d}\left(E_{F}\right)\left[=\int{ }^{E_{F}} n_{d}(E) d E\right]$ and $E_{\mathrm{B}}\left(E_{F}\right)\left[=\int{ }^{E_{F}} E n_{d}(E) d E\right]$ for various forms of $n_{d}(E)$ and numerically plotted the latter versus the former. (Only for the rectangular model can one directly get an analytical formula showing a strict, shifted, parabolic dependence $a\left[n_{d}\left(E_{F}\right)-5\right]^{2}$.) She used the mathematical formalism of moments (see Appendix A) to approximate the density of states (DOS) of the electrons in transition metals for two reasons: (i) it had already been used to approximate the DOS of the lattice vibrations by Montroll in 1942/3 [23], (ii) in the case of the frequency distribution of the normal states of a Hamiltonian describing a set of discrete atoms containing some degree of disorder, so that the cyclic boundary conditions are no more useful, physicists have found a way to calculate the moments by computing closed graphs, which one can also nicely visualize as closed walks, on the lattice. This was first found (published) by Pirenne in 1958 [24], and, independently at about the same time, by Domb, Maradudin, Montroll and Weiss in 1959 [25], for the study of vibrations on disordered systems (viz. crystals containing isotopes randomly distributed, or mixed binary alloys). In the case of Domb et al., the harmonic interactions were limited to first neighbour interactions (as it is the case in tight binding) and they could develop more applicable relations than Pirenne's general formulae. Thus this naturally was a method of choice for Fr. Cyrot-Lackmann (and Friedel) as she wanted to study the cohesion of liquid transition metals (after discussions with Loup Verlet who was working in the nearest neighbour university building in Orsay) [26]. It was also well suited to calculate surface tensions as there are simply fewer closed graphs related to surface atoms than to bulk atoms. 


\section{The Seventies (1970) and early Eighties; François Ducastelle and the second moment interaction model}

After computing the cohesive energy of a solid, the next idea naturally was to investigate its elastic properties. It was however rapidly realized that the simple rigid band model could not reproduce the bulk modulus (the lower the first nearest neighbour distance, the larger the $\beta$ overlap interaction, thus the larger the band and the larger the cohesive energy.) [27-28]. It was not possible to go further in a simple way with this band model in order to reproduce even just the main features of the experimental data collected by Gschneider Jr. for the equilibrium atomic volumes, the bulk moduli and the shear moduli which also exhibit parabolic shapes for the transition metal series, at least for the $4 d$ and $5 d$ series (Gschneider's figures 11, 6 and 3 respectively in his 1964 review paper [29]. The $3 d$ series is complicated by magnetism). François Ducastelle, Françoise Cyrot-Lackmann's thesis student, was the first to take this problem over by adding to the purely binding band contribution (purely binding in the simple model adopted) a purely repulsive contribution as a simple sum of repulsive pairwise interactions which he took of the Born-Mayer, $\exp (-p R)$, form, a form particularly suited to the exponential $R$-form (see Appendix C) of the overlapping integral $\beta$ chosen by Friedel at interatomic distances in the equilibrium condensed state (on the account that "their [small] number of spherical [nodes] allows them [the atomic $d$-orbitals] to decrease exponentially as $\exp (-q R)$ at [relatively short] distance from the origin" [30]). With respect to the usual all-pairwise potentials (e.g. Born, Lennard-Jones) it was important to clearly emphasize that the band binding contribution " $E_{\mathrm{B}}$ ne peut pas s'écrire comme une somme de contributions de paires d'atomes bien que $\mu_{2}$ s'obtienne de cette façon, ceci parce que $E_{B}$ est proportionnel à $\bigvee_{\mu_{2}}$ et non à $\mu_{2}$." [31]. With this natural and consistent choice of exponential functions, François Ducastelle could develop some relatively simple formulae for the equilibrium parameter, the total cohesive energy at equilibrium, the bulk modulus, and the cubic elastic shear moduli for various elementary atomic structures. For the fcc case for instance, he obtained $c_{44}$ and $c^{\prime}$ both proportional to 1-2q/p. François also obtained $\mathrm{c}_{44} / \mathrm{c}_{12}=(1-2 q / p) /(1-2 q / 3 p)$, and, in order to get a good agreement with experimental data, he indicated that $p$ should be comprised between $3 q$ and $5 q$. Thus, François Ducastelle could reproduce rather well, at least for the fcc transition metals, the non negligible departures from the Cauchy relationship for the cubic structures $\left(c_{12}=c_{44}\right.$, not to be confused with the $c^{\prime}=c_{44}$ isotropic relationship), a feature that a pairwise potential could absolutely not reproduce, 
whatever its parameterisation (for some comments on the Poisson-Cauchy relations, see Appendix D).

After reproducing the structural and cohesive properties of a solid at, and around its equilibrium state, the following step was to look after the properties of structural defects such as surfaces and vacancies (to start with): their energies of formation and their relaxed atomic structures. For instance it was known that the classical (non oscillatory) all-pairwise atomic potentials give a vacancy energy formation equals to the cohesive energy per atom, which contradicts experimental data for metals by a factor of about three to four, and that they predict an outward relaxation for surfaces when an inward relaxation is usually measured (much less was known about the atomic relaxations around a vacancy). The conceptual difficulty was, however, to go beyond the paradigm of periodicity, so connected to the paradigm of reciprocal space. It is easy to realize, afterwards, that the tight binding and moment approach is actually made for that. It proved difficult to realize it spontaneously at that time and this only came slowly. One had to be sure that no hypothesis or condition was violated, notably self-consistency.

In the manuscript of his thesis, Fr. Ducastelle showed that, because the number of $d$ electrons per atom in the solid does not change under a small elastic transformation, the formula $E_{\mathrm{B}}=$-const $\cdot \beta_{\mathrm{o}} \sqrt{\sum_{j} e^{-2 q R_{j}}}$ is valid for any filling of the $d$ band. In his article, however, he just wrote 'il est facile de voir' and did not even give that formula which, obviously for us now, can be re-written as $E_{\mathrm{B}}=$-const $\cdot \beta_{\mathrm{o}} e^{-q R} \sqrt{z_{c}}$ for a perfect, or only elastically deformed, crystalline structure, and to $E_{\mathrm{B}}(i)=-$ const $\cdot \beta_{\mathrm{o}} e^{-q R} \sqrt{z_{c}(i)}$ for every atom $i$ in a complex structural defect provided electrical neutrality is maintained at first order of approximation for each atom, which is true in metals thanks to metallic screening. It was simply beyond preoccupations in 1970, and even in the simple case of perfect crystal, the (already solved) problem was raised eighteen years later by Ackland, Finnis and Vitek about the Gaussian formulation [32]. The choice of a Gaussian $n_{d}(E)$, instead of the rectangular function originally used by Friedel in 1964, actually rendered the 'il est facile de voir' less straightforward than in the rectangular case where $E_{\mathrm{B}}=-\left[\left(n_{d}^{2}-10 n_{d}\right) / 20\right] \cdot|\beta| \sqrt{z_{c}}$ which obviously gives $E_{\mathrm{B}}=$-const $\cdot \beta \mid \sqrt{z_{c}}$ if $n_{d}$ remains constant per atom (see Appendix A). The choice of a Gaussian $n_{d}(E)$ came from the fact that it is the first level of the Edgeworth series which can naturally include higher order moments [22]. Within the second moment approximation, Ackland et al. [32] provided a mathematical demonstration of the validity of 
the proportionality to $\sqrt{\mu_{2}(i)}$, and thus to $\sqrt{z_{c}(i)}$, in any local environment which preserves the local charge neutrality and the original symmetry of the local DOS (only compression or stretching by a constant factor, plus global shift to maintain charge neutrality).

Considering electrical neutrality at the atomic level even around a vacancy also means neglecting the Friedel charge oscillations, which is entirely valid if one is not trying to analyse them, as we now know for sure. Might have it represented a conceptual difficulty for the students of Friedel in spite of Friedel's own pragmatism? It simply was not that obvious, and, whereas some people made it right in the details of their calculations, some people made it wrong. Sutton et al.'s article, with a first principles background, masterly settled the point in $1988[33]$.

Yet, in 1976, Allan \& Lannoo had already considered these problems, in an article which contains a discussion of the influence of self-consistency on the relaxation near a vacancy in transition metals [34]. They had also obtained a very simple formula for the fcc vacancy formation energy: $E_{\mathrm{vac}} \sim E_{\mathrm{coh}} \cdot(1-2 q / p) /(2[1-q / p])$ (to first order in $\left.1 / z_{\mathrm{c}}=1 / 12\right) . E_{\mathrm{coh}}$ is the corresponding cohesive energy, with respect to neutral free atoms), which gives a ratio $\mathrm{E}_{\mathrm{vac}} / \mathrm{E}_{\mathrm{coh}}$ comprised between 0.25 and 0.375 for $3 q \leq p \leq 5 q$, which sensibly agrees with experimental values (first nearest neighbour pair potentials give $\mathrm{E}_{\mathrm{vac}}=\mathrm{E}_{\mathrm{coh}}$ ).

Allan and Lannoo [34-36], Gordon, Cyrot-Lackmann and Desjonquères [37], Masuda and Sato [38], Gupta [39], Desjonquères and Spanjaard [40-41], Tomanek and Bennemann [42]: They all used Ducastelle's model to investigate more or less complex systems, including adatoms, small clusters, screw dislocation, carrying out some more or less extended relaxation on the atomic positions near the defect, always trying to keep closed form analytical formulae as much as possible. When it was not possible, as in the case of the screw dislocation in dilute bcc transition metal alloys, Masuda and Sato had everything to "calculate the total energy as a function of the atomic displacements" and "the atoms in the crystallite [were] relaxed along the screw dislocation." [38]

\section{1984; the Finnis-Sinclair article}

So what was new with Finnis and Sinclair 1984 [43], who acknowledged they chose " $f(\rho)$ to be $\sqrt{ }_{\rho}$, so as to mimic the result of tight-binding theory"? I see several important points (from $a$ to $f)$ :

a. The title: "A simple empirical N-body potential for transition metals." By contrast with previously mentioned articles, they do not announce the calculation of a specific property but 
they present a tool.

$b$. Their pragmatism: "the present approach is empirical", "the consequences of the form of the model (...) do not depend on the physical interpretation." One may compare this attitude with Newton's "hypotheses non fingo." Pragmatism also includes some amount of antiCartesianism.

c. A general program: "for the purpose of atomistic simulation", with explicit consideration for forces at the atomic level: "In order to perform atomistic simulations of crystals containing defects it is essential to have a rapid algorithm for calculating the force on each atom." They do provide the reader with general analytical formulae for calculation of forces. This will allow for Molecular Dynamics, not just static minimization. (On MD simulations using nbody potentials, see Appendix E.)

d. The Zeitgeist: Daw and Baskes had just published their two articles [44-45]. So it was in the air. In an interview in August 2000, Michele Parrinello declared that in 1983 "we invented what is called the glue potential, on which Ercolessi wrote his thesis. He was drafted for military service, and the work was stopped for a year and a half. During that time other people had similar ideas, and applied them in metals. So due to the draft we lost time, and bit of priority on this problem, but that's life." [46]

Yet there are obvious philosophical differences between these three approaches:

(i) Ercolessi, Parrinello and Tosatti declared that "although further theoretical justifications of this scheme can be found in the so-called embedded-atom method," they did "not believe that a truly first principles derivation can be given" [47] and they worked out purely empirically constructed analytical functions, carried out once, for gold [48].

(ii) Daw and Baskes used purely numerical functions deduced from first principle calculation or via a semi-empirical approach. These functions can only be tabulated and plotted.

(iii) Finnis and Sinclair used simple analytical functions, with the square root embedding function, "so as to mimic the result of tight-binding theory."

The origin of Daw and Baskes Embedded Atom Method has later been traced back, probably by Foiles [49-50], to Friedel 1952 impurity-in-jellium model [51] (see Appendix F). The basic idea from which Daw and Baskes drew on is that an atom in a metallic environment does not feel the detailed structure of the nearby atoms due to the effective screening in metallic systems. Each atom is considered as hosted in a homogeneous gas (LDA spirit) and its energy becomes a functional of the local host electron density in which it is immersed. With this approach which only considers the local electronic density, it is difficult to distinguish between fcc and hcp structures for instance. A pragmatic way to go is to consider 


\section{Acknowledgments}

I thank Professor Jacques Friedel, as well as François Ducastelle, for many invaluable discussions, by post mail and email. I also wish to thank the library department of the École Polytechnique for providing me with many ancient articles and books.

\section{Appendix A: the origins of the moments and the method of moments}

A known positive function (reasonably well behaved, e.g. a weight function, a probability distribution function) can be geometrically described (analysed) through its moments (which are usually normed and centred, but attention must be paid if a more general formalism is adopted). The zeroth order one corresponds to its surface (its mass, usually normed to 1), the first order moment gives the centre (centre of mass, the mean value of the weighted $X$-line), with the second order moment one can get a measure of the 'width' of the function (the root mean square deviation with respect to the chosen origin, directly the standard deviation if it is with respect to the centre of the function), with the third order moment combined with the 
previous one one can get a measure of the asymmetry of the function, and with the fourth order moment one can have some indication on the mono or bimodality of the function.

If one does not know the function by itself, but can have access to its first moments (one way or another), one then has methods to approximate that function although the approximation is not unique and may not be too good if one is after a precise description of the function (this is usually not the case at the beginning of a new field of research, as with Friedel in the $60 \mathrm{~s}$, but it rapidly turned out to be in the $80 \mathrm{~s}$ ). The expression of a distribution as a function of its moments does not constitute a converging series at all. When the number of moments gets large, an elegant solution to that so-called moment problem is based on the continued fraction technique. This is a mathematical problem which goes back to I.J. Bienaymé (1852) and P.L. Čebyšev who is the founder of the use of continued fractions for the method of moments (Tchebychef 1874, his disciple Markov in 1884, and Stieltjes, independently, in 1894). The solution is also closed to the recursive method (Lanczos' method, see [3-4], and all contributions by Heine, Haydock, etc., in [53]). For the historical connection between continued fractions and Padé approximants, see [54].

The 'second moment' is the second order moment, thus the third moment. As usual, beware of notations and conventions, normalisation (yes or not, per atom, or even per electronic state if they are all equivalent.) and choice of origin (e.g. centred or not).

With the following notation, $m_{n}=\int X^{n} f(X) d X ; \alpha_{n}=\frac{1}{m_{0}} \int X^{n} f(X) d X=\overline{X^{n}}$ and $\mu_{n}=\frac{1}{m_{0}} \int\left(X-\alpha_{1}\right)^{n} f(X) d X=\overline{x^{n}}$ with the centred variable $x=X-\alpha_{1}$, I simply recall the following classical results (e.g. see [55]):

\begin{tabular}{|l|l|l|l|l|}
\hline$\mu_{0}=1$ & $\mu_{1}=0$ & $\mu_{2}=\overline{x^{2}}$ & $\mu_{3}=\overline{x^{3}}$ & $\mu_{\mathrm{n}}=\overline{x^{n}}$ \\
\hline$\alpha_{0}=1$ & $\alpha_{1}=\bar{X}$ & $\alpha_{2}=\mu_{2}+\alpha_{1}^{2}=\mu_{2}+\bar{X}^{2}$ & $\alpha_{3}=\mu_{3}+3 \alpha_{1} \mu_{2}+\alpha_{1}^{3}$ & $\alpha_{\mathrm{n}}$ \\
\hline $\mathrm{m}_{0}=S$ & $\mathrm{~m}_{1}=S \alpha_{1}$ & $\mathrm{~m}_{2}=S \alpha_{2}$ & $\mathrm{~m}_{3}=S \alpha_{3}$ & $\mathrm{~m}_{\mathrm{n}}=S \alpha_{\mathrm{n}}$ \\
\hline
\end{tabular}

From the notation $\mu_{2}: \sigma^{2}$, one gets $\sigma^{2}=\alpha_{2}-\alpha_{1}^{2}=\bar{X}^{2}-\bar{X}^{2}$.

When the distribution is known, the $\mu_{n}$, because they are centred, are the easiest moments to calculate.

The second moment approximation does not mean that there is a series which is truncated to its second moment (or third term). It means that no other parameter is available. 
Yet, with only three moments, the distribution can be rectangular, Gaussian, parabolic, ... For instance let us consider a rectangular distribution of surface $S$, of width $w$ and centred on $M$, and a Gaussian (Laplace-Gauss) distribution of surface $S$, of half 'width' $\sigma$ at $1 / \sqrt{ }$ e height and centred on $M$. These two distributions being symmetric with respect to their centres $\mathrm{M}$, their odd centred moments are null. Their even moments $\mu_{2 n}$ are $w^{2 n} /\left[(2 n+1) 2^{2 n}\right]$ for the rectangular distribution, and $\sigma^{2 n}(2 n) ! /\left(2^{n} n !\right)$ for the Gaussian distribution. Thus nothing more than three parameters, $S, M$ and $w$, or $S, M$ and $\sigma$, are required to entirely describe these two distributions and their moments. Conversely, three parameters, $\mathrm{m}_{0}, \mathrm{~m}_{1}$ (or $\left.\alpha_{1}\right)$ and $\mathrm{m}_{2}$ (or $\alpha_{2}$ or $\mu_{2}$ ) can define a rectangular or a Gaussian distribution. These two distributions obviously have different shapes (and different moments beyond $\mu_{2}$, e.g. $\mu_{4}$ equals $144 \mu_{2}^{2} / 90$ for the rectangular case, and $3 \mu_{2}^{2}$ in the Gaussian case).

For an elementary crystal which can be described by a tight-binding Hamiltonian, one considers that every atom has $\mathrm{m}_{0}$ equivalent electronic state available (10 for the $d$ states, spin state included), and the $\{$ Pirenne-Domb et alii-Cyrot-Lackmann $\}$-theorem gives $\alpha_{1}=E_{0}$, the inner atomic level which is equal to the atomic level $E_{a t}$ plus a shift $\alpha$ due to the fact that the atoms are not isolated, and $\mu_{2}=z_{c} \beta^{2}$ (there are $z_{c}$ closed walks which go to a first neighbour and thus contribute, way and return, $\beta \cdot \beta$ ). Assuming a rectangular distribution, one thus gets $w^{2} / 12=\mathrm{z}_{\mathrm{c}} \beta^{2}$ hence $w=\sqrt{ }_{12} \sqrt{ }_{\mathrm{c}}|\beta|$ whereas $n_{d}\left(E_{F}\right)=\int_{E_{0}-w / 2}^{E_{\dot{b}}} A d E=\frac{10}{w}\left[e_{F}+w / 2\right]$ (since $S=A w=\mathrm{m}_{0}=10$ and noting $e$ the centred variable $\left.e=E-E_{0}\right) \quad$ and $E_{B}=\int_{E_{0}-w / 2}^{E_{E}}\left(E-E_{a t}\right) A d E \sim \frac{10}{2 w}\left[e_{F}^{2}-(w / 2)^{2}\right]$ if one neglects the shift $\alpha$ for the sake of clarity, which gives, after some simple algebraic manipulations, $E_{B}=-2.5 w+\frac{w}{20}\left[n_{d}\left(E_{F}\right)-5\right]^{2}$, a shifted parabola in $n_{d}\left(E_{F}\right)$, and also a linear function in $w$, hence $E_{\mathrm{B}}=$-const $\cdot|\beta| \sqrt{z_{c}}$. Assuming a Gaussian distribution does not alter the conclusions but makes the demonstration purely numerical for the parabolic shape, and more subtle (at least to my taste) for the linear dependence in $|\beta| \sqrt{z_{c}}$. In his truly remarkable sometimes intuitive way, Friedel 'knew' in 1964 that these results would not depend much on the shape of $n_{d}(E)$. Yet, as far as I am concerned, I can understand it only through the demonstrative work carried out by Fr. CyrotLackmann. 


\section{Note on the bimodality indicator}

Jean-Pierre Gaspard [3-4] noticed that the ratio $\tilde{b}=\left(\mu_{4} \mu_{2}-\mu_{3}^{2}-\mu_{2}^{3}\right) / \mu_{2}^{3}$, which can be deduced from the first two terms of the continued fraction of the resolvant of the distribution function, gives some indication on the mono or bimodality of a distribution. A distribution with $\tilde{b}>>1$ is monomodal whereas a distribution with $\tilde{b}<<1$ is bimodal. It is tempting to propose a critical value for $\tilde{b}$. However such a critical value does not exist in a universal way. Just to give two examples, it is 0.8 for the symmetrical beta law $\left([x(1-x)]^{a-1}\right.$, for $0 \leq x \leq 1, a$ being a real number $>0$ ) but for the symmetrical mixture of two equivalent Gaussians differing only by their centres, it is 1.5 . The case of a non symmetrical distribution will be more difficult to decide. Let us also note that, using Pearson's coefficients $\beta_{1}=\mu_{3}^{2} / \mu_{2}^{3}$ and $\beta_{2}=\mu_{4} / \mu_{2}^{2}$, one has $\tilde{b}=\beta_{2}-\beta_{1}-1$.

\section{Some original quotations which introduced the moments:}

Marin Mersenne (French monk and universal scientist, well known for being in touch with almost all savants of his time), in his free translation/adaptation in 1634 of the not yet published lectures of Galileo Galilei on Mechanics at the University of Padua in the 1590s: “Le moment eft l'inclination du mefme corps, lors qu'elle n'eft pas feulement confidérée dans ledit corps, mais conioinctement auec la fituation qu'il a fur le bras d'vn leuier, ou d'vne balance." [56]

Galileo Galilei, in Le Mecaniche, posthumously published in Ravenna in 1649: "Momento è la propensione di andare al basso, cagionata non tanto dalla gravità del mobile, quanto dalla disposizione che abbino tra di loro diversi corpi gravi" [57]

Irénée-Jules Bienaymé (1852): “(...) si l'on appelle $\mu_{\Sigma}$ la moyenne des puissances $\Sigma$ de toutes les erreurs possibles, on aura $\int \varepsilon^{\Sigma} d \varepsilon \varphi(\varepsilon)=\mu_{\Sigma}{ }^{\prime}[58]$

Pafnouty Lvovitch Tchebychev (1874): "En cherchant à tirer tout le parti possible sur les valeurs limites de l'intégrale $\int_{a}^{h} f(x) d x$ des valeurs des intégrales $\int_{A}^{R} f(x) d x, \int_{A}^{R} x f(x) d x$, $\int_{A}^{B} x^{2} f(x) d x, \int_{A}^{B} x^{m} f(x) d x$, où l'on a $A<a, B>b$, et où $f(x)$ reste positive, je suis parvenu à 


\section{Appendix B: on Tight Binding and LCAO}

Felix Bloch demonstrated (in much more details than some forerunners as Houston, Strutt, Frenkel, and quite independently) that if the ionised atoms are positioned regularly, as in a crystal, then, for the electrons in such a periodic lattice, there are solutions of Schrödinger's equation in the form of plane waves that describe electrons travelling in any direction through the crystal without being scattered [61]. The Bloch plane-wave solutions are more complicated than the de Broglie wave of an electron in free space, but a wave-vector $\boldsymbol{k}$ may still be defined: one has to deal with de Broglie waves, "die im Rhytmus des Gitteraufbaus moduliert sind." Exact solutions for any particular choice of the periodic potential $V(\boldsymbol{r})$ (: the interaction potential between the conduction electrons and the lattice) are probably impossible to find and Bloch worked out exact solutions in a limit case he knew from his time in Zürich where he, and Heitler and London enjoyed walking together. F. Bloch drew on the idea that Heitler and London had used on their treatment of the hydrogen molecule, that of constructing electron wave functions starting from a basis of unperturbed single-atom ground-state orbitals. F. Bloch called it the limit case of stark gebundener Elektronen (strongly bound electrons), which came to be know as tight-binding approximation. In his 1931 article, Erich Hückel extensively referenced F. Bloch [62]. At the request of their common director Heisenberg, Peierls, among many other things, worked out the solution for a nearly free version of $V(\boldsymbol{r})$, instead of the tight-binding version chosen by Bloch, and was delighted to get 
some similar results which were thus likely to be independent on the details of the interactions of the electrons with the atoms. A.H. Wilson developed the band theory further (see [63-65]).

Wigner and his student Seitz blurred the landscape in 1933 when they explicitly considered the work of Bloch as mainly based on the hypothesis of free electrons in chemistry [9]. Mott and Jones coined the English expression 'tight binding' approximation in their 1936 book [12]. Friedel and his co-workers always quoted Hückel for the tight-binding approximation.

Yet, in 1931, referring to F. Bloch 1928, Erich Hückel wrote: "Es lassen sich dann die Eigenfunktionen eines Elektrons durch Linearkombinationen der Eigenfunktionen des Elektrons bei getrennten Atomen annähern.” (p. 219 of [62]). And, in 1955, Reitz clearly wrote: "the linear combination of atomic orbitals, or Bloch, or tight binding method. This technique was first proposed for solids by Bloch.” (page 16 of [66])

It must be recognized that Bloch's name, because of Bloch's theorem, conveys the idea of periodicity, and Fourier transforms. By contrast, because Hückel dealt with finite size molecules, his name conveys the idea of as distorted as you like configurations with a real space approach (even if Hückel happened to also use Bloch's periodicity trick because he actually dealt with benzene, a cyclic 'aromatic' molecule!). It is also probably difficult to comprehend that some electrons may be delocalized without being totally free. The most relevant picture here is that of itinerant electrons, hopping from one atom to the next, but not necessarily forming a homogeneous jellium between the atoms as free electrons do.

Lennard-Jones is usually given as the founder of the LCAO method in 1929 [67] although this was the way of doing at that time: Hund 1927 onward, Mulliken 1928, LennardJones 1929. Mulliken formalized the technique and introduced the English term 'LCAO' in $1935[68]$.

There is a difference in spirit between Heitler and London 1927 and the so-called HundMulliken approach, at least in their premises. Heitler \& London 1927 start with atomic orbitals, AOs, (e.g. $\varphi_{\mathrm{A}}(\boldsymbol{r})$ and $\varphi_{\mathrm{B}}(\boldsymbol{r})$ ) which they combine, through multiplications and additions, to generate symmetrical and antisymmetrical functions. The building blocks of the Hund-Mulliken approach are LCAOs, sums and differences of atomic orbitals (e.g. $\varphi_{\mathrm{A}}(\boldsymbol{r})+\varphi_{\mathrm{B}}(\boldsymbol{r})$ and $\left.\varphi_{\mathrm{A}}(\boldsymbol{r})-\varphi_{\mathrm{B}}(\boldsymbol{r})\right)$ which are then combined to generate symmetrical and antisymmetrical functions. These building blocks are more appropriate when the atoms are relatively close together, yet still retaining some of their atomic character: this is the spirit of the 'tight binding' approach. 
Tight binding has also been successfully developed for covalent materials by Friedel, Leman, Lannoo, Allan, Gaspard, and others with the use of moments. We are dealing here with $s$ and $p$ electronic states, which are quite different in nature from the atomic like $d$ states. But because of the $s p^{3}$ hybridization, these materials crystallize in the cubic diamond atomic structure, which is an open structure so that the influence of the second neighbours can be neglected in a first approximation with respect to the influence of the first neighbours. A tight binding treatment is relevant, and successful. The electrons are nevertheless free to move from one atom to any other through the covalent bounds and the $E(k)$ dispersion for silicon is very close to the free electron dispersion at small $k$. The cubic diamond materials have been the first for which n-body potentials have been developed [69], although for a completely different reason. The tetrahedral angles between the $s p^{3}$ bonds is a fundamental of the cubic diamond structure and can only be reproduced with three body terms with explicit angular dependence (and it takes three points to define an angle). The Cauchy relations are violated for cubic diamond materials for two reasons: the interactions are not pairwise and the atoms are not centres of symmetry. The Cauchy discrepancy being negative, an embedded function of the usual type cannot be expected to work. The presence of explicit angular dependent terms seems necessary.

\section{Appendix C: exponential or power laws?}

The exponential form of the overlap, or transfer or hopping or bond, integral, has been chosen historically, in part because of the widespread use of the "Slater" orbitals [70]. Ab initio calculations that the dependence should rather be of the inverse power form [71-72].

In the immediate vicinity of the equilibrium first neighbour distance $R_{0}$, it does not matter since one has $\mathrm{e}^{-\kappa R} \sim c R^{-n}$ with $n=\kappa R_{o}$ and $c=\left(R_{o} / \mathrm{e}\right)^{n}$. If one wants to stretch the bonds further, i.e. to extend the validity of the overlap law, both solutions fail and pragmatism must prevail, especially for the smoothing of the function down to zero (e.g. see $\$ 7.3 .2$ of $[8])$. 


\section{Appendix D: on the Cauchy relations}

Following the mechanical school of Navier, Poisson, Cauchy, Clausius and Saint-Venant, one should have special relations between elastic constants which A.E.H. Love called Cauchy relations in the historical introduction of his famous treatise on elasticity more than a century ago [73]. These relations are consequences of the hypothesis of interactions between atomic point centres, together with other hypotheses such as pairwise interactions, atoms being symmetry centres of the structure, and no constraints at zero deformation. They proved to be incompatible with experimental measurements (once properly made..., for instance by W. Voigt) so that the atomic hypothesis was contested by positivist anti atomists such as Pierre Duhem, by no means an obscurantist. He was right in the sense that the real atoms are not point centres with no internal structure. From an opposite point of view, in the sixth volume of the Handbuch der Physik series in 1928, J.W. Geckeler wrote that for the elementary metals which violate the Cauchy relations, one must conclude that their atomic structure is not just a simple (Bravais) lattice. Still later on, Raman and his disciples in India, and Laval in France were to oppose Born about the number of independent elastic constants for diamond.

Cauchy's name is also associated to the opposite mechanical school, viz. the continuous mechanics tradition. The appellation Poisson-Cauchy relations would be more fitted, from an historical point of view.

Daw and Baskes noted that the positive curvature of the embedding function implies positive Cauchy discrepancies. Finnis and Sinclair reproduced the same remark, noting that it is the case for the square root function. Thus for metals with negative discrepancies, such as rhodium and iridium, it means that these metals have some basic properties which are not taken into account by the EAM model, for instance explicit angular dependant terms (hence the MEAMs: modified EAMs).

DFT ab initio calculations, in full electrons or with pseudopotentials, only started in the eighties, with sometimes only a moderate success as far as the calculation of elastic constants is concerned (see [1]). 


\section{Appendix E: the first MD simulations with n-body potentials}

As far as I know, the first MD simulation with a metallic n-body potential was carried out by S.M. Foiles in 1985 with an EAM potential for liquid transition and noble fcc metals (Ni, Pd, $\mathrm{Pt}, \mathrm{Cu}, \mathrm{Ag}$, and $\mathrm{Au})$ [74].

It is worth noting that at the same time, Stillinger and Weber designed an n-body potential for silicon, with explicit angular dependence, based on experimental and ab initio data, and also performed Molecular Dynamics calculations to check the liquid state [75].

Ercolessi, Parrinello and Tosatti in 1988 (published) performed MD simulations with their glue gold model in order to check the melting temperature of their model [52].

Rosato, Guillope and Legrand in 1989 are the first who carried out MD simulations with FS-like potentials, with Ducastelle's exponential functions, on fcc transition metals [76].

For sake of completeness, I also mention the MD simulations of systems of rigid molecules: When atoms of rigid molecules interact together through relatively long ranged Van der Waals interactions, one can say that the molecules, which are the elementary (rigid) bodies of the system, interact together through n-body potentials. The first MD simulation of this kind is by Barojas, Levesque and Quentrec in 1973 [77]. The interaction model was described in 1967 by Sweet and Steele [78].

For a short introduction to atomistic simulations, Monte Carlo Metropolis and Molecular Dynamics, with some historical information, see [79].

\section{Appendix F: on the origins of the EAM}

Stephen Foiles wrote in 1996 ([50], + see [49]) that "the idea of an embedding energy was first suggested by Friedel [51] and later developed further by Nørskov and Lang [effective medium theory 1980] and by Stott and Zaremba [quasi-atom concept 1980].”

In the late $40 \mathrm{~s}$, J. Friedel carried out the first numerical study of grain boundaries at the atomistic level (see [80]). Yet, a little disappointed by the crudeness of the pairwise interatomic potentials he had had to use, he decided to learn more about electronic interactions in metals and was introduced by his cousin Ch. Crussard to N.F. Mott in Bristol. There he also met Charles Frank, and his future wife.

In 1936, Mott, concerned with dilute solid solutions (metallic alloys, Hume-Rothery 
electron concentration rule (the famous 21/13 ratio, in 1926), influence on electric resistivity) had considered the problem of local screening of charges around (highly dispersed) impurities in a metallic matrix, notably when the dissolved and solvent metals do not occupy the same column in the periodic table, e.g. when an atom of zinc or aluminium is dissolved in copper. "The extra electrons of the dissolved atom must be regarded as belonging to the lattice rather than to the atom. We should therefore expect the dissolved atom to carry an extra positive charge $z$, equal to 1 for zinc, 2 for aluminium" [81]. Friedel ([51, 82]) modified Mott's rapidly decaying solution (à la Debye-Hückel) into a less rapidly decaying solution with oscillations which later came to be known as 'Friedel oscillations'.

The conceptual difficulty master was to consider the extra electrons of the impurity as given to the lattice, thus unbound, and yet to have the jellium respond as a local charge concentration (electron polarization cloud). One indeed finds in Friedel 1952 the idea that "an impurity atom is embedded in a regular lattice", and that the perturbation is only local (then, self consistently, that embedded atom can only depend on its local environment).

It must however be recognized that the lack of simple guidelines, except complex ab initio calculations or optimization fitting on empirical data, is not in the spirit of J. Friedel's approach.

\section{Appendix G: unabridged references}

[1] O.B.M. Hardouin Duparc, Copper and Venus. How copper atoms interact, in Copper, edited by J.M. Welter (Wiley-VCH, Weinheim, 2006) pp.18-24.

[2] G. Allan, Elementary electronic structures in semiconductors and transition metals, in Electronic structure of crystal defects and of disordered systems, Summer School Aussois Sept. 1980, edited by Fr. Gautier, M. Gerl and P. Guyot (Éditions de Physique, Les Ulis, 1981) pp.3-44.

[3] J.-P. Gaspard, Atomic and Electronic Structures of Amorphous Systems, in Electronic Structure of Crystal Defects and of Disordered Systems, Aussois 1980, edited by Fr. Gautier, M. Gerl and P. Guyot (Éditions de Physique, Les Ulis, 1986) pp.279-317.

[4] J.-P. Gaspard, Du cristal à l'amorphe : structure et cohésion, in Structures et Instabilités, Beg-Rohu 1985, edited by C. Godrèche (Éditions de Physique, Les Ulis, 1986) pp.441-544. 
[5] F. Ducastelle, Order and Phase Stability in Alloys (North-Holland, Amsterdam, 1991).

[6] A. Sutton, Electronic Structure of Materials (Clarendon Press, Oxford, 1993).

[7] D. Pettifor, Bonding and Structures of Molecules and Solids (Clarendon Press, Oxford, 1995).

[8] M. Finnis, Interatomic forces in Condensed Matter (Oxford Univ. Press, Oxford, 2003).

[9] E. Wigner and F. Seitz, On the Constitution of Metallic Sodium, Phys. Rev. 43 (1933) pp.804-810.

[10] N.F. Mott, A Discussion of the Transition Metals on the Basis of Quantum Mechanics. Proc. Phys. Soc. 47 (1935) pp.571-588.

[11] H. Fröhlich, Elektronentheorie der Metalle (J. Springer Verlag, Berlin, 1936).

[12] N.F. Mott and H. Jones, The Theory of the Properties of Metals and Alloys (Clarendon Press, Oxford, 1936).

[13] F. Seitz and R.P. Johnson, Modern Theory of Solids, J. Applied Physics 8 (1937) pp.84$97+$ pp.186-199.

[14] E. Wigner and F. Seitz, Qualitative Analysis of the Cohesion in Metals, in Solid State Physics, edited by F. Seitz and D. Turnbull, 1 (1955) pp.97-126.

[15] J.C. Slater, The ferromagnetism of nickel, Phys. Rev. 49 (1936) pp.537-545.

[16] J.C. Slater, Wave Functions in a Periodic Potential, Phys. Rev. 51 (1937) pp.846-851.

[17] J.C. Philips, Band Theory of Transition Metals, in Theory of Magnetism in Transition Metals, Proc. 1966 Varenna (Enrico Fermi) Summer School, Course 37, edited by W. Marshall (Academic Press, New York, 1967) pp.22-49.

[18] J. Friedel, Ferromagnetic Transition Alloys, in Theory of Magnetism in Transition Metals, Proc. 1966 Varenna (Enrico Fermi) Summer School, Course 37, edited by W. Marshall (Academic Press, New York, 1967) pp.283-318.

[19] N.F. Mott, Récents progrès et difficultés de la théorie électronique des métaux, in Ferromagnétisme, volume II of Le magnétisme, International Conference on Magnetism, Strasbourg, May 1939 (CNRS-IICI, Paris, 1940) pp.1-39 (with discussions).

[20] L. Néel, Champs moléculaires, aimantation à saturation et constantes de Curie des éléments de transition et de leurs alliages, in Ferromagnétisme, volume II of Le magnétisme, International Conference on Magnetism, Strasbourg, May 1939 (CNRS-IICI, Paris, 1940) pp.65-164 (with discussions).

[21] J. Friedel, On the Possible Impact of Quantum Mechanics on Physical Metallurgy, Transactions of the Metallurgical Society of AIME 230 (1964) pp.616-632. 
[22] F. Cyrot-Lackmann, Sur le calcul de la cohésion et de la tension superficielle des métaux par une méthode de liaisons fortes, J. Phys. Chem. Solids 29 (1968) pp.1235-1243.

[23] E.W. Montroll, Frequency Spectrum of Crystalline Solids, Journal of Chemical Physics 10 (1942) pp.218-229, 11 (1943) pp.481-495.

[24] Jean Pirenne, Dynamics of a crystal lattice containing isotopes, Physica 24 (1958) pp.7392.

[25] C. Domb, A.A. Maradudin, E.W. Montroll and G.H. Weiss, Vibration Frequency Spectra of Disordered Lattices. I. Moments of the Spectra for Disordered Linear Chains, Phys. Rev. 115 (1959) pp.18-24.

[26] F. Cyrot-Lackmann, On the Electronic structure of Liquid Transitional Metals, Advances in Physics 16 (1967) pp.393-400.

[27] S. Barisic, J. Labbé and F. Cyrot-Lakmann, Anomalies élastiques associées aux singularités de Van Hove de la densité d'états électronique d'une bande $s$ étroite, Journal de physique 30 (1969) pp.955-965.

[28] F. Ducastelle, personal communication, 2009.

[29] K.A. Gschneider Jr., Physical Properties and Interrelationships of Metallic and Semimetallic Elements, in Solid State Physics, edited by F. Seitz and D. Turnbull, 16 (1964) pp.275-426.

[30] J. Friedel, Transition metals. Electronic structure of the d-band. Its role in the crystalline and magnetic structures, in The Physics of Metals. 1. Electrons, edited by J.M. Ziman as a tribute to Sir N.F. Mott (Cambridge Univ. Press, Cambridge, 1969) pp.340-408.

[31] F. Ducastelle, Modules élastiques des métaux de transition, Journal de Physique 31 (1970) pp.1055-1062.

[32] G.J. Ackland, M.W. Finnis and V. Vitek, Validity of the second moment tight-binding model, J. Phys. F 18 (1988) pp.L153-L157.

[33] A.P. Sutton, M.W. Finnis, D.G. Pettifor and Y. Ohta, The tight-binding bond model, J. Phys. C 21 (1988) pp.35-66.

[34] G. Allan and M. Lannoo, Vacancies in Transition Metals. Formation energy and Formation volume, J. Phys. Chem. Solids 37 (1976) pp.699-709.

[35] G. Allan and M. Lannoo, Relaxation near Transition Metal Surfaces, Surf. Sci. 40 (1973) pp.375-387.

[36] G. Allan and M. Lannoo, Influence of Self-Consistency on the Relaxation near a Vacancy or a Surface in Transition Metals, Phys. Status Solidi (b) 74 (1976) pp.409-414. 
[37] M.B. Gordon, F. Cyrot-Lackmann and M.C. Desjonquères, Relaxation and stability of small transition metal particles, Surf. Sci. 80 (1979) pp.159-164.

[38] K. Masuda and A. Sato, Electronic theory for screw dislocation motion in dilute b.c.c. transition metal alloys, Phil. Mag. A 44 (1981) pp.799-814.

[39] R.J. Gupta, Lattice relaxation at a metal surface, Phys. Rev. B 23 (1981) pp.6265-6270.

[40] M.C. Desjonquères and D. Spanjaard, A simple chemisorption theory and its application to transition adatoms on transition metals, J. Phys. C 15 (1982) pp.4007-4021.

[41] D. Spanjaard and M.C. Desjonquères, Comment on "Universal features of bonding in metals", Phys. Rev. B 30 (1984) pp.4822-4827.

[42] D. Tomanek and K.H. Bennemann, simple theory for the electronic and atomic structure of small clusters, Phys. Rev. B 28 (1983) pp.665-673. (+ erratum 1984).

[43] M. Finnis and J.F. Sinclair, A simple empirical N-body potential for transition metals, Phil. Mag. A 50 (1984) pp.45-55.

[44] M.S. Daw and M.I. Baskes, Semiempirical, Quantum Mechanical Calculation of Hydrogen Embrittlement in Metals, P.R.L. 50 (1983) pp.1285-1289.

[45] M.S. Daw and M.I. Baskes, Embedded-atom method: Derivation and application to impurities, surfaces, and other defects in metals, Phys. Rev. B 29 (1984) pp.6443-6453.

[46] M. Parrinello, Interview by Dónal Mac Kernan, Simu-Newsletter 2 (2000) pp.5-14.

[47] F. Ercolessi, E. Tosatti, M. Parrinello, Au(100) Surface Reconstruction, P.R.L. 57 (1986) pp. 719-722.

[48] F. Ercolessi, M. Parrinello, E. Tosatti, Simulation of Gold in the glue model, Phil. Mag. A 58 (1988) pp. 213-226.

[49] M.S. Daw, S.M. Foiles, M.I. Baskes, The embedded-atom method: a review of theory and applications, Mater. Sci. Rep. 9 (1993) pp.251-310.

[50] S.M. Foiles, Embedded-Atom and Related Methods for Modeling Metallic Systems, MRS Bulletin 21\#2 (1996) pp. 24-28.

[51] J. Friedel, The Distribution of Electrons Round Impurities in Monovalent Metals, Phil. Mag. 43 (1952) pp.153-189.

[52] G.J. Ackland, G. Tichy, V. Vitek and M.W. Finnis, Simple N-body potentials for the noble metals and nickel, Phil. Mag. A 56 (1987) 735-756.

[53] The Recursion Method and its Applications, Imperial College Sept. 1984, Proceedings edited by D.G. Pettifor and D.L. Weaire (Springer, Berlin, 1985).

[54] Cl. Brezinski, The long History of Continued Fractions and Padé Approximants (Springer, Berlin, 1991). 
[55] G. Udny Yule, An Introduction to the Theory of Statistics (Ch. Griffin, London 1911, + enlarged and revised versions with M.G. Kendall).

[56] M. Mersenne, Les Mechaniques de Galilée Florentin, Ingenieur et Mathematicien du Duc de Florence, traduites de l'italien par le Père Marin Mersenne, Minime, avec plusieurs additions de lui-même (Henry Guenon, Paris, 1634).

[57] G. Galilei, Le Mechaniche (Ravenna 1649), for instance in Le opere di Galileo Galilei, vol. 2, (Ediz. Nazionale, Florence, 1891) pp. 147-191.

[58] I.J. Bienaymé, Mémoire sur les probabilités des erreurs d'après la méthode des moindres carrés, J. Mathématiques Pures et Appliquées, Ière série 17 (1852) pp.33-78.

[59] P. Tchebychef, Sur les valeurs limites des intégrales, Journal des mathématiques pures et appliquées 19 (1874) pp.157-160.

[60] Th.-J. Stieltjes, Recherches sur les fractions continues, Annales de la Faculté des Sciences de l'Université de Toulouse pour les sciences mathématiques et physiques, $1^{\text {ère }}$ série, 8 (1894) pp.1-122 (+ (1895) pp.1-64).

[61] F. Bloch, Über die Quantenmechanik der Elektronen in Kristallgittern, Zeitschrift für Physik 52 (1928) pp.555-600.

[62] E. Hückel, Quantentheoretische Beiträge zum Benzolproblem, Zeitschrift für Physik 70 (1931) pp.204-286.

[63] Sir Nevill Mott et al., The Beginnings of Solid State Physics, Proc. Royal Society London A 371 (1980) pp.1-177.

[64] L. Hoddeson, G. Baym and M. Eckert, The development of the Quantum Mechanical Electron Theory of Metals, 1926-1933, in Out of the Crystal Maze, edited by L. Hoddeson, E. Braun and S. Weart (Oxford Univ. Press, Oxford, 1992) pp.88-111.

[65] Sir Brian Pippard, Electron in Solids, in Twentieth Century Physics, edited by L.M. Brown, A. Pais and Sir Brian Pippard, vol. III (IOP, Bristol, 1995) pp.1279-1383.

[66] J.R. Reitz, Methods of the One-Electron Theory of Solids, in Solid State Physics, edited by F. Seitz and D. Turnbull, 1 (1955) pp.1-95.

[67] J.E. Lennard-Jones, The Electronic Structure of Some Diatomic Molecules, Trans. Faraday Soc. 25 (1929) pp.668-686.

[68] R.S. Mulliken, in Electronic Structures of Polyatomic Molecules and Valence. VI. On the Method of Molecular Orbitals, J. Chem. Phys. 3 (1935) pp.375-378.

[69] P.N. Keating, Effect of Invariance Requirements on the Elastic Strain Energy of Crystals with Applications to Diamonds, Phys. Rev. 145 (1966) pp.637-645.

[70] J.C. Slater, Atomic shielding constants, Phys. Rev. 36 (1930) pp.57-64. 
[71] V. Heine, s-d Interactions in Transition Metals, Phys. Rev. 153 (1967) pp. 673-682.

[72] W.A. Harrison, Electronic Structure and the Properties of Solids, (W.H. Freeman and Co., San Francisco, 1980).

[73] A.E.H. Love, A treatise on the mathematical theory of elasticity, (Univ. Press, Cambridge, 1892 1st edition).

[74] S.M. Foiles, Application of the embedded-atom method to liquid transition metals, Phys. Rev. B 32 (1985) pp.3409-3415.

[75] F.H. Stillinger and Th.A. Weber, Computer simulation of local order in condensed phases of silicon, Phys. Rev. B 31 (1985) pp.5262-5271.

[76] V. Rosato, M. Guillope and B. Legrand, Thermodynamical and structural properties of f.c.c. transition metals using a simple tight-binding model, Phil. Mag. A 59 (1989) pp.321336.

[77] J. Barojas, D. Levesque and B. Quentrec, Simulations of Diatomic Homonuclear Liquids, Phys. Rev. A 7 (1973) pp.1092-1104.

[78] J.R. Sweet and W.A. Steele, Statistical Mechanics of Linear Molecules. I. Potential Energy Functions, J. Chem. Phys. 47 (1967) pp.3022-3028.

[79] O.B.M. Hardouin Duparc, Atomistic Simulation, in Computer Simulation in Materials Science, NATO ASI Oléron 2005, edited by H. Kirchner, L. Kubin and V. Pontikis, (Kluwer, Dordrecht, 1996) pp.63-83.

[80] O.B.M. Hardouin Duparc, Charles Crussard's early contributions: Recrystallization in situ and a Grain Boundary study with J. Friedel and B. Cullity, Int. J. Mat. Res. 10 (2009) to be published.

[81] N.F. Mott, The Electrical Resistance of Dilute Solid Solutions, Proc. Camb. Phil. Soc. 32 (1936) pp.281-290.

[82] J. Friedel, Electronic Structure of Primary Solid Solutions in Metals, Advances in Physics 3 (1954) pp.446-507. 


\section{On the origins of the Finnis-Sinclair potentials}

Olivier Hardouin Duparc

LSI, CEA-X-CNRS, UMR 7642, École Polytechnique, 91128 Palaiseau Cedex, France

\section{Contents:}

\section{Introduction}

2. The Thirties; first steps, nullified by sensible hesitations

3. The Sixties; the time of Jacques Friedel and his $d$ band model

4. The Seventies (1970) and early Eighties; François Ducastelle and his followers

5. 1984; the Finnis-Sinclair article

6. Short conclusion; for a continuing story

Acknowledgments

Appendix A: the origins of the moments and the method of moments

Appendix B: on Tight Binding and LCAO

Appendix C: exponential or power laws?

Appendix D: on the Cauchy relations

Appendix E: the first MD simulations with n-body potentials

Appendix F: on the origins of the EAM

Appendix G: unabridged references 
Dear Professor Bristowe,

I thank you for your positive comment about my article.

I just took some vacations and that is why I am answering so late.

It is of course always difficult to translate texts which are some times very old (old French for Mersenne, old Italian for Galilei). Here are my tentative translations. Let me know if they are understandable (I hope I do not use false friends). Feel free to modify accordingly. Thanks.

In the attached FSOrigins_OHD-3.doc Word document, I embedded these translations in parentheses. (foot notes are not customary in Phil. Mag. Another appendix within the appendices would be cumbersome.)

" $E_{B}$ ne peut pas s'écrire comme une somme de contributions de paires d'atomes bien que $\mu_{2}$ s'obtienne de cette façon, ceci parce que $E_{B}$ est proportionnel à $\sqrt{ } \mu_{2}$ et non à $\mu_{2}$."

" $E_{B}$ cannot be written as a sum of atomic pair contributions although it is the case for $\mu_{2}$, because $E_{B}$ is proportional to $\sqrt{ } \mu_{2}$, not to $\mu_{2}$."

"Le moment eft l'inclination du mefme corps, lors qu'elle n'eft pas feulement confidérée dans ledit corps, mais conioinctement auec la fituation qu'il a fur le bras d'vn leuier, ou d'vne balance." [56]

"Moment is the inclination of the same body, when it is considered not only within the body, but with respect to the effect it has on the branch of a lever, or of a balance." [56]

"Momento è la propensione di andare al basso, cagionata non tanto dalla gravità del mobile, quanto dalla disposizione che abbino tra di loro diversi corpi gravi” [57]

"Moment is the propension to go down, considered not as much with respect to the weight of the solid, but rather to the relative disposition of the various weighting bodies" [57]

I am asking an Italian colleague about the correctness of this translation.

“(...) si l'on appelle $\mu_{\Sigma}$ la moyenne des puissances $\Sigma$ de toutes les erreurs possibles, on aura $\int \varepsilon^{\Sigma} d \varepsilon \varphi(\varepsilon)=\mu_{\Sigma} "[58]$

"(...) if one calls $\mu_{\Sigma}$ the average of the powers $\Sigma$ of all the possible errors, one will get $\int \varepsilon^{\Sigma} d \varepsilon \varphi(\varepsilon)=\mu_{\Sigma} "[58]$

"En cherchant à tirer tout le parti possible sur les valeurs limites de l'intégrale $\int_{a}^{h} f(x) d x$ des valeurs des intégrales $\int_{A}^{R} f(x) d x, \int_{A}^{B} x f(x) d x, \int_{A}^{B} x^{2} f(x) d x, \int_{A}^{R} x^{m} f(x) d x$, où l'on a $A<a, B>b$, 
et où $f(x)$ reste positive, je suis parvenu à reconnaître que ces recherches conduisent à des théorèmes d'un nouveau genre, concernant le développement de l'expression $\int_{A}^{R} \frac{f(x)}{z-x} d x$ en fraction continue, qui joue un si grand rôle dans la théorie des séries.” [59]

"Trying to learn as much as possible on the limiting values of the $\int_{a}^{b} f(x) d x$ integral from the values of the $\int_{A}^{B} f(x) d x, \int_{A}^{B} x f(x) d x, \int_{A}^{B} x^{2} f(x) d x, \int_{A}^{B} x^{m} f(x) d x$ integrals, where $A<a, B>b$, and where $f(x)$ remains positive, I realized that these researches lead to theorems of a new kind, concerning the developement of $\int_{A}^{R} \frac{f(x)}{z-x} d x$ in continued fraction, which plays such a great rôle in the theory of series." [59]

"Considérons sur une droite infinie une distribution de masse (positive), la masse $m_{i}$ se trouvant concentrée à la distance $\xi_{i}$ de l'origine. La somme $\Sigma m_{i} \xi_{i}^{k}$ peut être appelée le moment d'ordre $k$ de la masse par rapport à l'origine." [60]

"Let us consider an infinite straight line with a (positive) mass distribution, the mass $m_{i}$ being concentrated at a distance $\xi_{i}$ from the origin. The sum $\Sigma m_{i} \xi_{i}^{k}$ may be called the moment of order $k$ of the mass with respect to theorigin." [60]

"die im Rhytmus des Gitteraufbaus moduliert sind."

"which are modulated by the rhythm of the lattice structure."

"Es lassen sich dann die Eigenfunktionen eines Elektrons durch Linearkombinationen der Eigenfunktionen des Elektrons bei getrennten Atomen annähern."

"Thus the eigenfunctions of an electron can be approximated as linear combinations of eigenfunctions of atomic electrons."

I am asking a German colleague about the correctness of this translation.

p. 4 "For the Gaussian d-band, the width (support) is infinite ..". What is support?

The 'support' of a function $f(\mathrm{X})$ is the ensemble of $\mathrm{X}$ values over which $f(\mathrm{X})$ is finite (not zero). For a Gaussian, it goes from minus infinity to plus infinity. It is not what one usually calls the width of a Gaussian $(\sigma=\sqrt{ }$ var $)$.

p. 4 "nearest neighbour building" means "neighbouring building"?

Yes. (but neighbouring as 'nearest neighbour', I believe, although I have to check with Jacques Friedel. This is a petty detail)

p. 7 "made it right" means "got it right"?

Yes. Or "made it the rigth way", "made it correctly".

p. 10 "yes or not" means "whether or not"?

Yes.

I thank you for your help,

Olivier Hardouin Duparc. 